\title{
Response Dynamics and Directional Properties of Nonspiking Local Interneurons in the Cockroach Cercal System
}

\author{
Y. Kondoh, T. Arima, J. Okuma, and Y. Hasegawa \\ Honda R\&D Co. Ltd., Wako Research Center, Wako, Saitama 351-01, Japan
}

The response properties and directional receptive fields of nonspiking local interneurons in the cercal system of the cockroach are described. Wind-evoked responses were recorded intracellularly, and then analyzed by means of the Wiener kernel method in which a Gaussian white noise signal was used as a stimulus. Cross-correlation between the response and the white noise signal produced first- (linear) and second-order (nonlinear) kernels that were used to define input-output characteristics of the interneurons.

Three sets of interneurons were distinguished on the basis of kernel analysis. First, responses in interneurons 101, 107, 111 , and 203 were characterized predominantly by a differentiating, first-order kernel, which suggests a linear relationship to the stimulus. The amplitude and waveform of the kernel changed with the change in stimulus angle, indicating that these four cells are directionally sensitive. Second, responses in interneurons 102 and 103 were also directionally sensitive but highly nonlinear. The first-order kernel was biphasic, whereas the second-order kernel had an elongated depolarizing peak on the diagonal. The response dynamics were accounted for by a cascade of two filters, a linear band-pass filter and a static nonlinear filter, in which the nonlinearity is a signal compression (or a rectification). Third, responses in interneurons 104 and 201 consist largely of the second-order nonlinear component. The second-order kernel, which had an elongated depolarizing peak or a hyperpolarizing valley on the diagonal, did not show any directional preference. The second-order nonlinearity was dynamic, and could be modeled by a band-pass linear filter-static nonlinearity-low-pass linear filter cascade, where the static nonlinearity is a full-wave rectification. The band-pass filter would simply reflect the mechanical property of cercal hair sensilla, whereas the low-pass filter represents the transfer at synapses between the cercal afferents and the interneurons.

The nonlinear response thus explains the difference in the directional sensitivity while the differentiating first-order kernel explains the velocity sensitivity of the interneurons. We show that 101 and 107 respond most preferentially to wind from the left versus right, whereas 102, 103, 111, and 203 respond to wind from the front versus rear. Thus, it is sug-

\footnotetext{
Received Apr. 6, 1992; revised Sept. 21, 1992; accepted Nov. 2, 1992.

We are grateful to Prof. K.-I. Naka and Dr. P. Newland for their valuable comments on the manuscripts, and to M. Nasu for his help in computation. We also thank F. Takahashi for his encouragement.

Correspondence should be addressed to Dr. Yasuhiro Kondoh, Honda R\&D Co. Itd., Wako Research Center, Chuo 1-4-1, Wako, Saitama 351-01, Japan.

Copyright (C) 1993 Society for Neuroscience $0270-6474 / 93 / 132287-19 \$ 05.00 / 0$
}

gested that there are two subsystems responding maximally to wind displacement along two coordinate directions, one for the longitudinal direction and the other for the transverse direction. On the other hand, the full-wave-rectifier nonlinear interneurons are omnidirectional, and thus suggested to code simply the power of the wind displacement.

[Key words: nonspiking interneurons, white noise analysis, cross-correlation, directional coding, cercal system, cockroach]

Only in a few systems have the dynamics of the neuronal elements been examined to model neural coding and nonlinear processing of mechanosensory signals, and in the insects these have concentrated mainly on wind-sensitive hairs on the terminal abdominal appendages, the cerci. Air disturbance or wind is the essential cue for eliciting escape behavior in the cockroach (Camhi, 1980). It is initially detected by filiform hairs on the cerci, which have preferred directions of deflection such that the sensory neurons are excited by movement in only one direction (Dagan and Camhi, 1979; Westin, 1979). The sensory neurons make direct connections with a number of intersegmental interneurons, some of which have large-diameter axons, referred as cercal giant interneurons (GIs), and are involved in escape behavior (Camhi and Tom, 1978; Ritzmann and Camhi, 1978; Camhi, 1980; Camhi and Nolen, 1981). The velocity and directional sensitivities to wind stimuli of the cercal GIs have been extensively studied, and were estimated by analyzing the responses to a step or sinusoidal changes in wind velocity in terms of the normalized postsynaptic potential arca, the spike frequency (or the poststimulus time histogram), and the spike threshold (cricket: Bacon and Murphey, 1984; Kanou and Shimozawa, 1984; Jacobs et al., 1986; Miller et al., 1991; cockroach: Daley and Camhi, 1988; Camhi and Levy, 1989). In such conventional analyses, less attention has been directed to the dynamics of the cellular processes to encode the cercal input. For example, no consideration has been taken into account for properly evaluating the cercal input, particularly inhibition. Most of these studies, therefore, have determined the receptive fields of the interneurons only in terms of excitatory, or simply the most optimal directions.

A popular method used to test the dynamic response of a system, whether or not it is physiological, is to examine its response to an impulse or a step. If a system is linear, its impulse response allows us to predict the system's response to any arbitrary input. It is, however, not always possible to obtain a system's impulse response, especially in many mechanosensory systems. Sinusoidal modulation of the stimulus was therefore used most often in these systems, although the mechanosensory 
signal naturally varies in a chaotic or a white noise-like fashion for an individual neuron. Wiener kernel analysis using Gaussian white noise as the stimulus is a powerful method by which to examine quantitatively the dynamic responses in such a system. The Gaussian white noise signal contains, as well as an impulse, every possible frequency and all amplitudes, with a Gaussian distribution of signal amplitude, making it the most effective stimulus. Using this analysis, a cellular response is decomposed into a series of orthogonal functionals (Wiener kernels) or filters that are produced by cross-correlation of a generalized input, Gaussian white noise, and the response (Marmarelis and Naka, 1972; Marmarelis and Marmarelis, 1978; Naka et al., 1985). In the cockroach cercal system, we have shown that slow potential (graded) response in a wind-sensitive interneuron of the terminal ganglion can be modeled by a differentiating first-order kernel, suggesting that the response is linearly related to the stimulus wind, and sensitive to the wind velocity (Kondoh et al., 1991a). Computing the kernels by cross-correlation between the wind stimulus and a spike train of the cercal afferents, we have also shown that the cercal receptors act as band-pass filter with peak frequencies of $106 \mathrm{~Hz}$ (Kondoh et al., 1991b). The afferents are thought to be velocity sensitive because of the differential, firstorder kernel. In the present article, we characterize the response dynamics (frequency and gain characteristics) and directional sensitivities of several wind-sensitive, nonspiking local interneurons in the cockroach terminal ganglion by means of the Wiener kernel method.

A survey based on intracellular recording and staining has uncovered a great number of wind-sensitive, local interneurons in the cockroach terminal ganglion (Kondoh et al., 1989) as well as in the cricket (Kobashi and Yamaguchi, 1984; Bodnar et al., 1991 ) and the locust (Boyan et al., 1989). Some of the local interneurons are nonspiking, whereas others produce spikes. Of special interest are the nonspiking interneurons that are shown here to be involved in processing of information from the primary cercal afferents, whereas such nonspiking local interneurons are usually involved in the control and coordination of motor output in insect segmental ganglia (Siegler, 1984). They normally respond to wind in a graded manner without producing spikes, and are thereby particularly suited to this systematic analysis because the spike process is a highly nonlinear event. From the kernel analysis emerge three sets of interneurons, one linear and two nonlinear types. We show that the linear interneurons are characterized mainly by differentiating first-order kernels, suggesting they are velocity sensitive. The nonlinear types are modeled by a cascade of a band-pass linear filter, a static nonlinear filter (either half-wave or full-wave rectification), and then a low-pass linear filter (LNL filter cascade). The linear and half-wave rectifier interneurons are directionally sensitive, and are suggested to respond maximally to wind displacement along one of two coordinate directions, the longitudinal or transverse one.

\section{Materials and Methods}

Preparation. We used adult male cockroaches, Periplaneta americana, taken from a laboratory colony in all experiments. For physiological experiments, we followed the procedure described previously (Kondoh et al., 1991a). Briefly, animals were pinned, dorsal side up, to a corkcovered platform and fixed with beeswax. After exposing the ventral nerve cord and the terminal ganglion, the body cavity was flooded with standard cockroach saline. To provide the appropriate tension to the ganglion, a silver platform was introduced posteriorly between the right and left cercal nerves to support it. A bipolar silver hook electrode placed under the cercal nerve was used to stimulate the cercal afferents electrically.

Stimulation and recordings. Details of the method for stimulating the interneuron by Gaussian white noise and a sinusoidal wave are given in our previous article (Kondoh et al., 1991a). The stimulus wind was delivered to the animal through a plastic tube $10 \mathrm{~cm}$ in length and 5 $\mathrm{cm}$ in diameter; the other end of the tube was closed by a loudspeaker with a diameter of $10 \mathrm{~cm}$. The stimulus angle was varied by rotating the wind tube about the specimen in the horizontal plane. The direction, intensity, and phase of air displacement were calibrated with respect to the loudspeaker voltage by means of a displacement transducer attached t 5 the surface of the loudspeaker moving plate. The rising phase of the source signal corresponds to a push of the moving plate toward the specimen, and the falling phase corresponds to a pull.

Intracellular recordings were usually made from the neuropilar segment of interneurons during stimulation by Gaussian white noise or sinusoidal modulation of wind. A glass micropipette filled with $0.1 \mathrm{M}$ Lucifer yellow in $0.1 \mathrm{M} \mathrm{LiCl}$ at the tip and with $2 \mathrm{M} \mathrm{KCl}$ at the shaft served as an intracellular recording electrode. Electrodes with DC resistance of $150-200 \mathrm{M} \Omega$ were used. The mean resting potential obtained for some of the interneurons ranged from -37.7 to $-46.2 \mathrm{mV}$ under this condition. For later analysis, stimulus and cell response were stored on magnetic tape using an FM data recorder (A-65, Sony Magnescale Inc.). They were shown to be nonspiking according to a set of physiological criteria established in locust ganglia by Burrows and Siegler (1976). After the recording of neuronal activity, neurons were stained by iontophoretical injection of Lucifer yellow ( $-5 \mathrm{nA}$ for $2-5 \mathrm{~min})$. The filled ganglia were then dissected out, frxed in $10 \%$ formalin in saline, dehydrated in alcohol series, and cleared in methyl benzoate. The dyefilled cell was viewed on a fluorescence microscope and drawn using a drawing tube.

Analysis of the response. Cellular responses recorded were first fed to a 16-bit personal computer (PC-9800VX, NEC, Tokyo, Japan) through a 12-bit analog-to-digital converter (ADX-98H, Canopus Electronics Corp.) at a sampling rate of $2 \mathrm{kHz}$ for $10-20$ sec. Data were then transferred to a VAXstation 3100 computer (Digital Equipment Corp.) on which software for white noise analysis, STAR, was run. The firstand second-order kernels were used to identify the dynamics of the system whose input was the movement of a loudspeaker's moving plate, and whose output was cell response. For computation of kernels and convolutions, we followed the technique performed on the slow potentials in catfish retinal cells described by Sakuranaga and Naka $(1985 a, b)$. The first-order kernel is the first-order cross-correlation between the input, a Gaussian modulation of wind stimulus, and the output, cellular response. The kernel is the best linear approximation of a system's impulse response. Therefore, if a system is linear, its impulse response can predict the system's response to any arbitrary input. The secondorder kernel is a function of two time lags, $\tau_{1}$ and $\tau_{2}$, and defines the multiplicative interaction between two impulse responses. The magnitude of the nonlinear response is shown on the third dimension with the contour lines in a two-dimensional plot. In figures, solid lines indicate positive or depolarizing peaks, and broken lines, negative or hyperpolarizing valleys. The kernel unit for the first-order kernel is $\mathrm{mV} \cdot \mu \mathrm{m}^{-1} \cdot \mathrm{sec}^{-1}$, and that for the second-order kernel is $\mathrm{mV} \cdot \mu \mathrm{m}^{-2} \cdot \mathrm{sec}^{-2}$ for slow potential response.

Models were predicted by convolving white noise input with the firstand second-order kernels. Discrepancy between the response and model (kernel prediction) was represented by a mean square error (MSE) between the response and model. The MSE is given as a percentage by computing the ratio of the deviation between the response and the model, and indicates the accuracy of the model prediction by the kernels. For example, the MSE for the linear model represents the degree of linearity, that is, the ratio of a linear component in a total response. Detailed algorithms for computing the kernels, model responses, power spectra, and MSEs have been described in Sakuranaga and Naka (1985a) and Sakai and Naka (1987).

Classification of interneurons. There is a relatively large number of local and intersegmental interneurons involved in the cercal system of the cockroach terminal ganglion. Thus, we designated the interneuron by a three-digit number similar to the designation adopted in the thoracic interneurons of locust (Robertson and Pearson, 1983) and cockroach (Westin et al., 1988). The first numeral indicates the course of axons in the anterior connective and the location of cell body and dendritic fields. The two subsequent numerals specify the interneuron within the class. The general classes are 101-199 for the bilateral type 


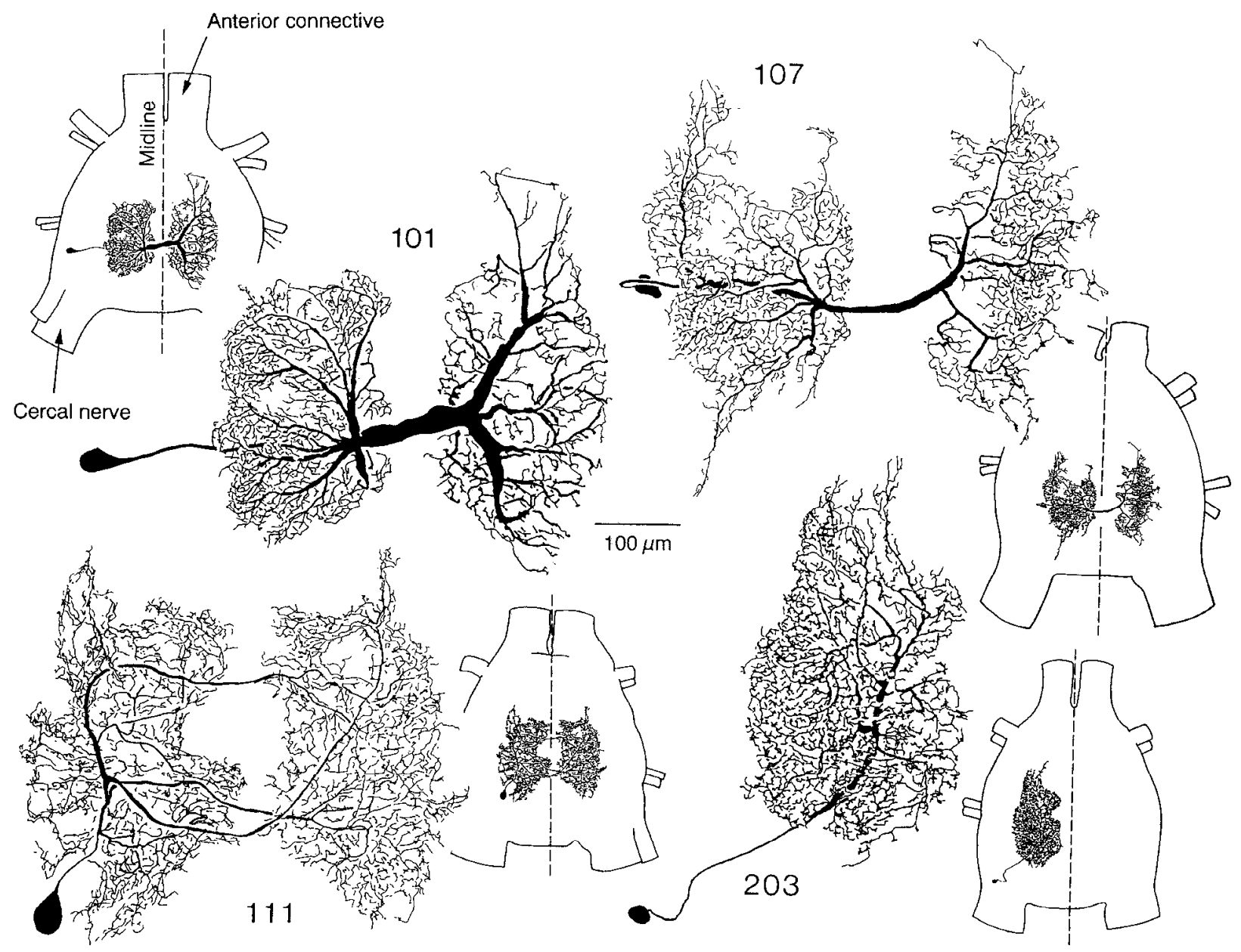

Figure 1. Morphology of a group of interneurons whose wind-evoked responses were linearly related to stimuli. The drawings are of a wholemount of the terminal ganglion, viewed dorsally and with anterior at the top. The smaller drawings show the location of the interneuron within the contours of the ganglion. The broken line indicates the midline of the ganglion.

of local interneurons, 201-299 for the unilateral type of local interneurons with dendritic fields ipsilateral to the cell body, and 301-399 for the unilateral type of local interneurons with dendritic fields contralateral to the cell body. The first numbers of our cells, representing the general classes, do not match those given by Westin et al. (1988). This is because the terminal ganglion lacks the intersegmental interneurons with a descending axon. Thus, the same digit number of a neuron in the terminal abdominal ganglion and in the thoracic ganglion does not indicate that they are serially homologous.

\section{Results}

\section{Interneurons 101, 107, 111, and 203}

The nine uniquely identifiable interneurons examined in this study were categorized into three physiological types on the basis of kernel analysis. The first type comprises interneurons 101, 107,111 , and 203 , in which the wind-evoked response was found to be linear, and characterized by a differentiating firstorder kernel. Figure 1 shows the morphology of this set of interneurons. Interneurons 101 and 107 resemble each other in morphology as well as in physiology. Both have a small cell body (approximately $20-30 \mu \mathrm{m}$ in diameter) lying in the ventrolateral cortex of the ganglion and two dendritic arborizations on either side. However, the transverse neurite that interconnects the bilateral dendritic fields is $30-40 \mu \mathrm{m}$ in diameter for 101 , much thicker than that of 107 (about $10 \mu \mathrm{m}$ in diameter).
Interneuron 111 has a cell body $(40-50 \mu \mathrm{m}$ in diameter) on the dorsolateral cortex of the ganglion, and three transverse neurites intervening the dendritic fields on both sides of the ganglion. On the other hand, 203 has a $20-\mu \mathrm{m}$-diameter cell body near the base of the cercal nerve and dendritic branches only on the side ipsilateral to the cell body.

Figure 2 shows polar representations of first- and secondorder kernels in 101, 107, 111, and 203 at various angles. Responses to white noise evoked in 101 and 107 were almost the same with respect to their kernel configurations and directional properties. Around the long-axis or midline of the animal, the polarity of the first-order kernel was reversed (Fig. $2 A, B$ ). Wind stimuli directed at ipsilateral (ipsi) $90^{\circ}$ and contralateral (cont) $90^{\circ}$ had the largest amplitude and were roughly mirror images of each other; the ipsilateral stimulus produced an initial depolarization, whereas the contralateral stimulus produced an initial hyperpolarization. Thus, the linear response evoked on the opposite side of the animal's body is equivalent to that evoked by a polarity-inverted stimulus. This is to be expected from a linear system. The mean peak time of the first-order kernel ranges from $6.1 \mathrm{msec}$ to $6.7 \mathrm{msec}(n=10)$ for 101 , and from $7.3 \mathrm{msec}$ to $7.6 \mathrm{msec}(n=3)$ for 107 , in preferred directions. The waveform of the first-order kernel is approximated by a time differential of a brief pulse, indicating that the linear part 
Figure 2. Polar representations of the first- and second-order kernels in 101, 107,111 , and 203 , showing the directional selectivity of the first-order kernel. The two kernels identify the dynamics of a system whose input is the movement of a loudspeaker's moving plate, and whose output is the cell's response. The second-order kernel is produced by the nonlinear interaction between two impulse responses, and thereby has two time axes. The magnitude of nonlinear response is shown on the z-axis as a contour. Depolarizing peaks are shown by solid lines and hyperpolarizing valleys by broken lines. Orientation of the stimulus is in relation to the body axis of the animal body. For example, $0^{\circ}$ represents wind stimulus delivered posteriorly along the long axis of the animal's body, and $180^{\circ}$ represents wind delivered anteriorly. Ipsi $90^{\circ}$ represents wind stimulus perpendicular to the long axis and ipsilateral to the cell body of the interneuron, and Cont $90^{\circ}$ represents wind delivered to the cell body. Left column, The firstorder kernels obtained from three or four different animals were superimposed. Note that the waveform of the kernels is reversed in polarity at ipsi $60^{\circ}$ and cont $120^{\circ}$ in 111 and 302 , and at $0^{\circ}$ and $180^{\circ}$ in 101 and 107 . Right column, The second-order kernels. 1st-order kernel

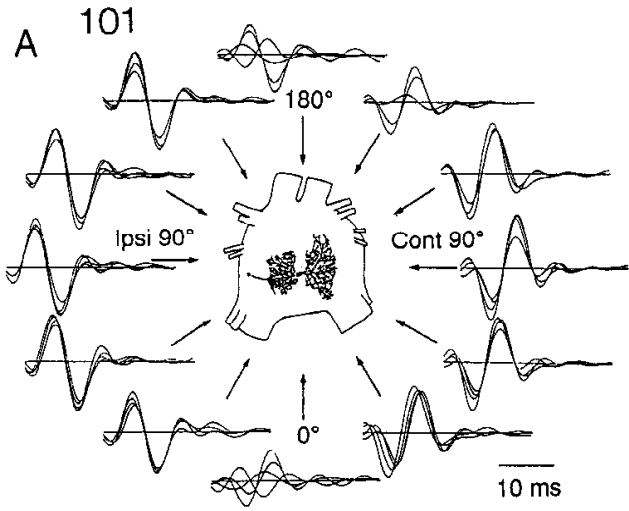

107
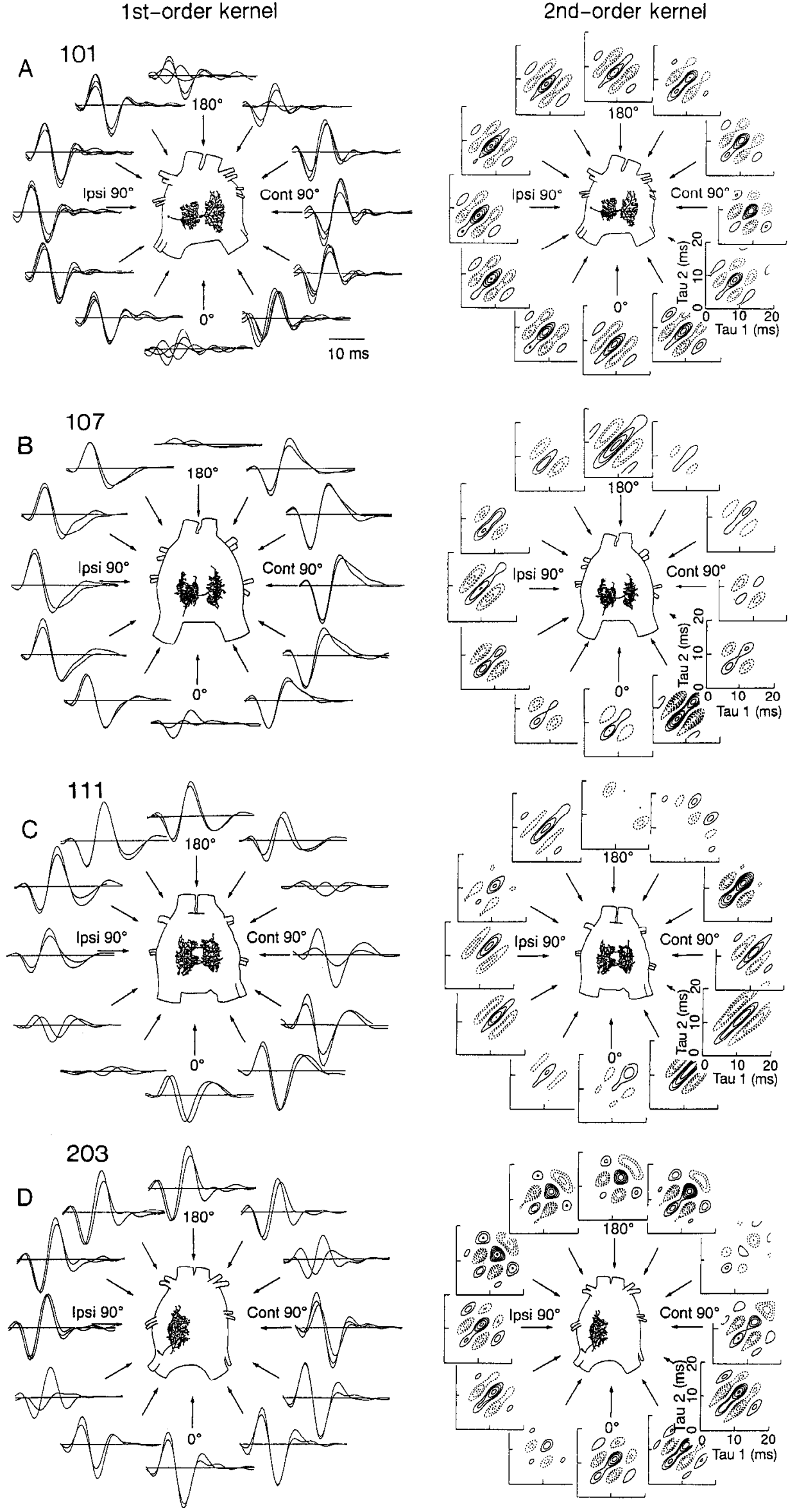
A1 Interneuron 107
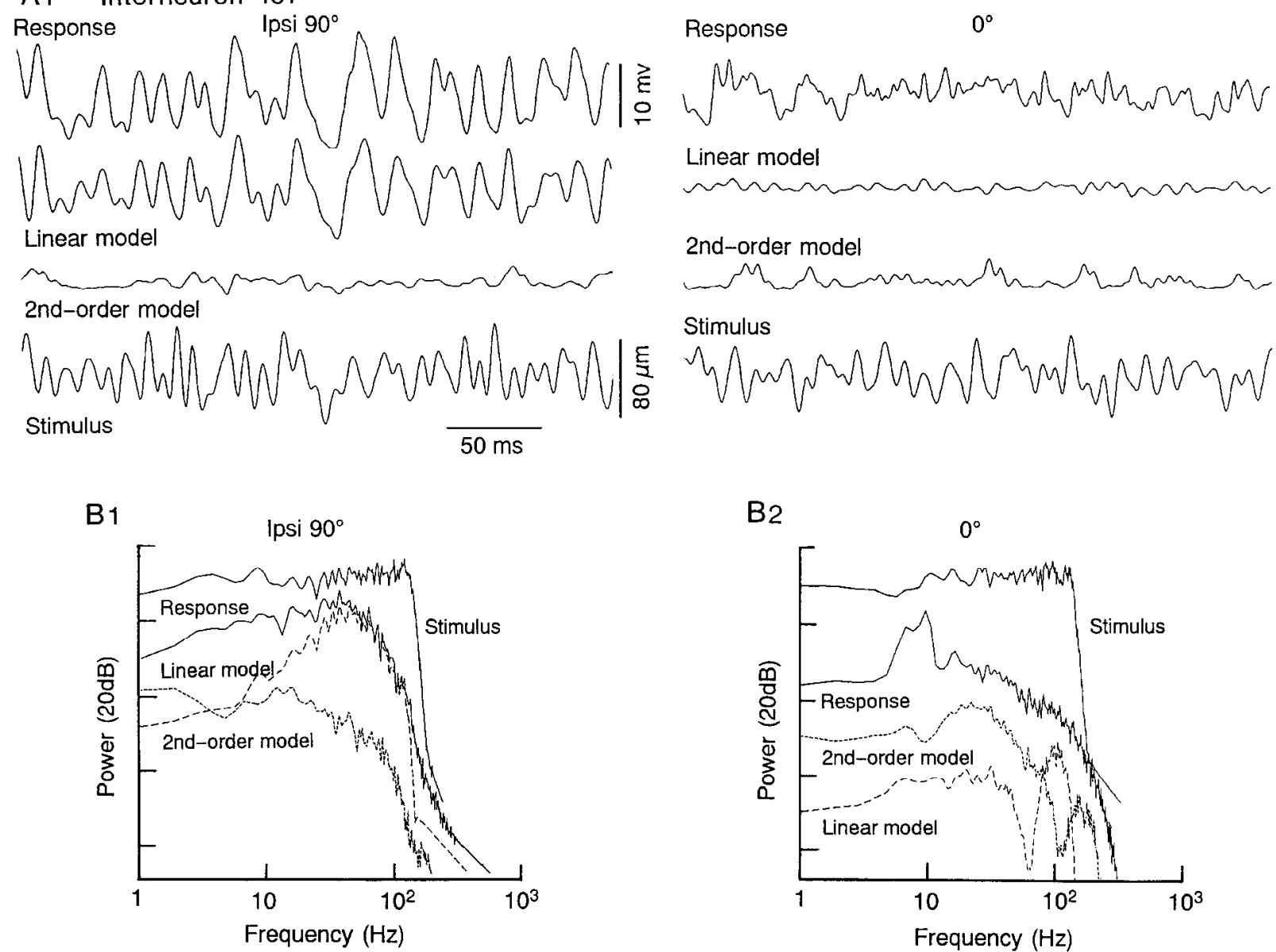

Figure 3. A, Intracellular recordings from 107 evoked by white noise modulation of wind (Stimulus) at ipsi $90^{\circ}\left(A_{1}\right)$ and $0^{\circ}\left(A_{2}\right)$, and the linear and second-order nonlinear models. When stimulated at ipsi $90^{\circ}$, the linear (first-order) model predicted the actual response quite well. MSEs for the linear and second-order model were $28.2 \%$ and $23.7 \%$, respectively. Thus, the linear component comprises a major part of the total response, whereas the second-order nonlinear component comprises a very small part, as it improves the MSE only by $4.5 \%$. At $0^{\circ}$, the response was significantly small. MSEs for the linear and second-order nonlinear models were $93.6 \%$ and $75.3 \%$, respectively. $B$, Frequency responses in 107 , showing power spectra of the input white noise (Stimulus) and cellular responses; the kernel predicted models are shown for ipsi $90^{\circ}\left(B_{l}\right)$ and $0^{\circ}$ $\left(B_{2}\right)$ stimulus.

of response in 101 and 107 is a differential of sensory input, that is, a wind-velocity wave. The function of these interneurons as seen by its linear component is to detect the wind velocity. The similar waveform of kernels at all stimulus angles (except their polarity), shows that although the amplitude (gain) of the linear component differed, the frequency characteristics were invariable irrespective of the stimulus angle.

The first-order kernels in 111 and 203 were also differentiating (Fig. 2C,D). In both interneurons, the first-order kernels were largest in amplitude when the wind stimulus was at $0^{\circ}$, ipsi $150^{\circ}$, cont $30^{\circ}$, and $180^{\circ}$, that is, when the wind was almost longitudinal to the animal's long axis. They were very small or noisy when the wind stimulus was orthogonal but somehow diagonal to the animal's long axis. The polarity of the kernels reversed at ipsi $60^{\circ}$ and cont $120^{\circ}$. These were null directions. The kernels were initially hyperpolarized when stimulated at ipsi $120^{\circ}$, whereas they showed initial depolarization when stimulated at cont $60^{\circ}$. The time to the peak ranged from 8.3 to $8.5 \mathrm{msec}$ ( $n$ $=2$ ) for 111 and from $7.3 \mathrm{msec}$ to $7.8 \mathrm{msec}$ for $203(n=3)$ in the preferred directions. Thus, the peak time of the first-order kernel in 111 was slightly longer than that in 101,107, and 203, suggesting that the response in 111 was slower.

The second-order kernels in this set of interneurons were not distinctive. The kernel configurations were considerably different from angle to angle: some kernels had an elongated depolarizing peak on the diagonal, whereas the others had only offdiagonal valleys (Fig. 2). This was because the second-order nonlinear component was very small as indicated by the large MSEs of the second-order model. Figure 3 shows the responses and models to the white noise stimulus in 107 for two different stimulus angles, one for a preferred (ipsi $90^{\circ}$ ) and the other for a null direction $\left(0^{\circ}\right)$, which were orthogonal to each other. At ipsi $90^{\circ}$, the linear (first-order) component (or model) was large and reasonably similar to the response with an MSE of $28.2 \%$, whereas the second-order component was much smaller (Fig. $3 A_{l}$ ). MSE for the second-order model was $23.7 \%$. As MSE for the second-order model was computed between the summation of the two models and the actual response, the linear and second- 
A1 Interneuron 111

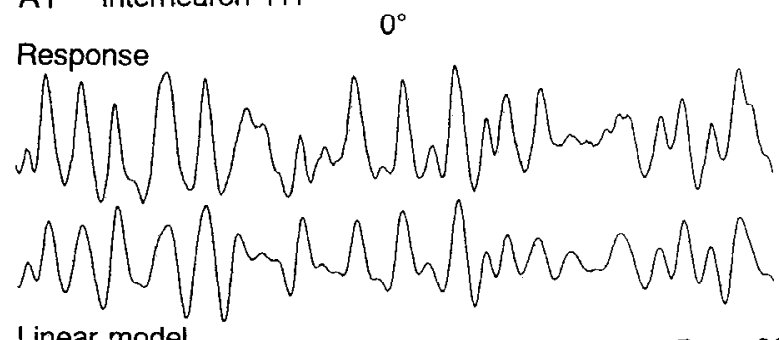

Linear model

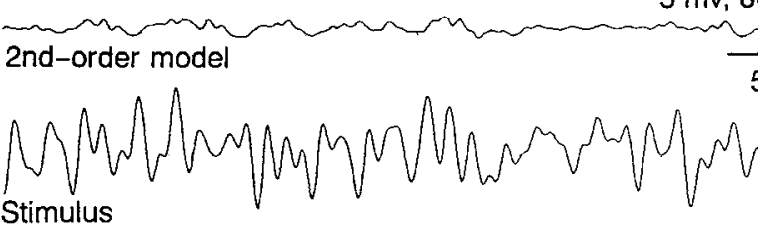

A2

Ipsi $60^{\circ}$

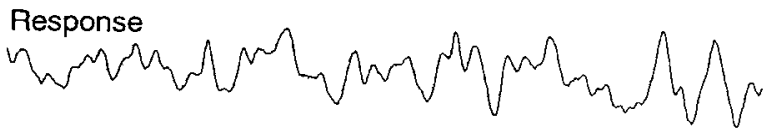

Linear model

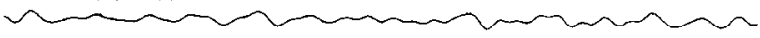

2nd-order model

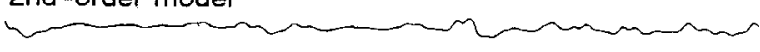

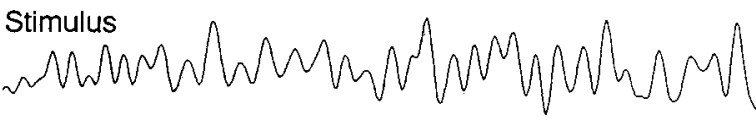
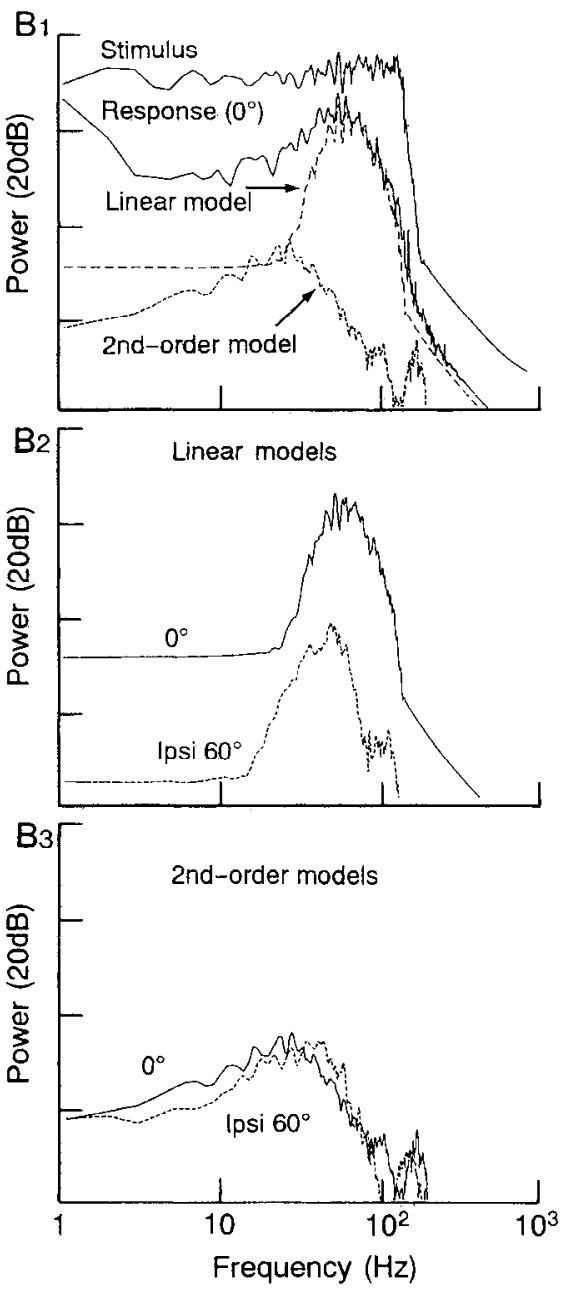

Figure 4. $A$, Intracellular recordings from 111 evoked by white noise stimuli at $0^{\circ}\left(A_{1}\right)$ and ipsi $60^{\circ}\left(A_{2}\right)$ corresponding first-order (linear) and second-order nonlinear models. In a preferred direction $\left(0^{\circ}\right)$, the linear model makes a good match with the actual response (the MSE was $37.8 \%$ ). MSE for the second-order model was $35.4 \%$. At ipsi $60^{\circ}$, MSEs for the linear and second-order model were $94.7 \%$ and $87.1 \%$, respectively. $B$, Frequency responses in 111 , showing power spectra of the input white noise $\left(B_{l}\right)$, cellular response $\left(B_{l}\right)$, and kernel predicted models for $0^{\circ}$ and ipsi $60^{\circ}$ stimuli $\left(B_{2}\right.$ and $\left.B_{3}\right)$.

order nonlinear components were thought to constitute $71.8 \%$ and $4.5 \%$ of the total response, respectively. Those parts of the response not predicted by the linear or second-order nonlinear components were due to noise or a higher-order nonlinear component. At $0^{\circ}$, the linear model was very small (Fig. $3 A_{2}$ ). This confirmed our observation that the first-order kernel was very small and noisy when wind was delivered orthogonally to the long axis of the animal's body (Fig. $2 B$ ). Analyses of the response in the frequency domain confirm the observations we made on the kernels and the predictions. The power spectrum of the response from 107 evoked at ipsi $90^{\circ}$ always had a peak at $40-$ $50 \mathrm{~Hz}$ (Fig. $3 B_{l}$ ). The linear model was very sharply band-passed with a peak at $70-90 \mathrm{~Hz}$, and had power comparable to that of the responses in a higher-frequency region, whereas the secondorder nonlinear component was much smaller. It was significantly reduced when stimulated at $0^{\circ}$ (Fig. $3 B_{2}$ ).

Figure 4 shows another example of responses to white noise and the corresponding model predictions, which was from 111 at the stimulus angles of $0^{\circ}$ and ipsi $60^{\circ}$. In contrast to 101 and 107 , the linear component for the $0^{\circ}$ stimulation was large and reasonably similar to the actual response (Fig. $4 A_{1}$ ), while the second-order nonlinear component was much smaller (Fig. 4 $A_{2}$ ). MSE for the models suggested that the linear component pre- dicted $62.2 \%$ of the total response, whereas the second-order model predicted only $2.4 \%$ of a total response. The power spectra of the response at $0^{\circ}$ and the linear models had a peak at about $70 \mathrm{~Hz}$ (Fig. 4B, ), indicating that the response and linear component are band-passed. The power of the linear model was comparable to that of the response around the peak, indicating that the high-frequency response of 111 is almost linear. In a null direction (ipsi $60^{\circ}$ ), neither the linear nor the second-order nonlinear model could predict the response (Fig. $4 A_{2}$ ). The MSE for the second-order model was at most $87.1 \%$. The linear model at ipsi $60^{\circ}$ had a power of $20-40 \mathrm{~dB}$ less than that evoked at $0^{\circ}$ (Fig. $4 B_{2}$ ). This confirmed our observation that for the ipsi $60^{\circ}$ stimulus, the interneuron produced no significant response. In both directions, the second-order nonlinear component had a power of $20 \mathrm{~dB}$ below that for the actual response (Fig. $4 B_{3}$ ).

\section{Interneurons 102 and 103}

The second type comprises interneurons 102 and 103 whose wind-evoked responses were also directionally sensitive but highly nonlinear. Both interneurons have a cell body with a diameter of $30-40 \mu \mathrm{m}$ in the dorsal cortex of the ganglion (Fig. 5 ). Near the midline of the ganglion, the primary neurite loops ventrally to run into the ventral neuropil, where it increases its 


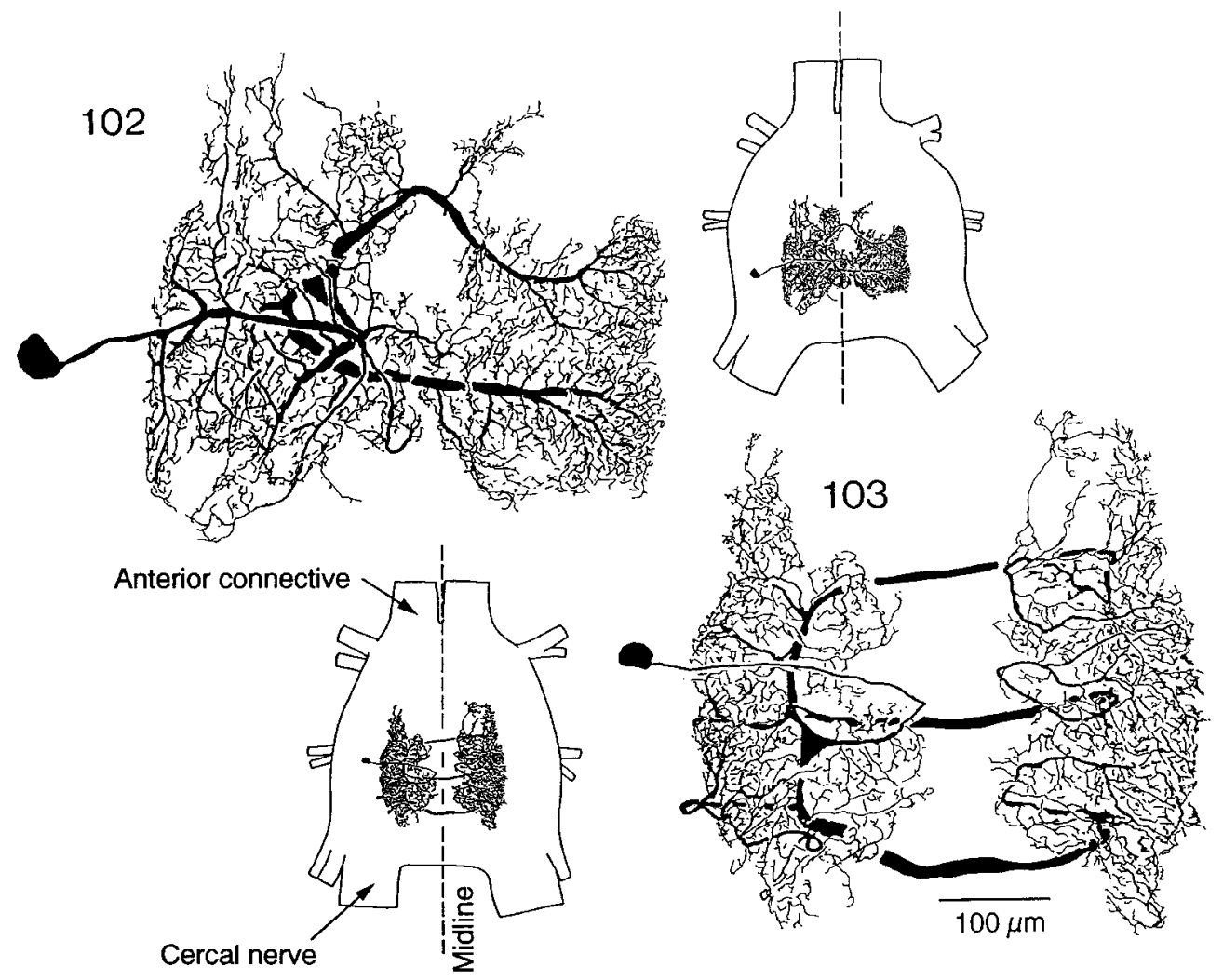

Figure 5. Morphology of a group of nonlinear interneurons, viewed dorsally. Broken line indicates the midline of the ganglion. Anterior is to the top. diameter up to $10-20 \mu \mathrm{m}$. In 102 , the main neurite bifurcates into two thick neurites, which then turn medially to form a "C"'-shaped structure and run into the contralateral neuropil, where they give rise to numerous finer processes. By contrast, 103 has three thick transverse neurites (20-30 $\mu \mathrm{m}$ in diameter) that run parallel to each other near the ventral surface of the ganglion and connect the bilateral arborizations.

Interneurons 102 and 103 produced well-defined first-order kernels that were biphasic with the initial depolarizing or hyperpolarizing phase, as shown in Figure 6 . The time to the peak, ranging from $6.9 \mathrm{msec}$ to $7.4 \mathrm{msec}(n=4)$ for 102 and from $6.8 \mathrm{msec}$ to $7.1 \mathrm{msec}(n=8)$ for 103 , was relatively constant irrespective of the stimulating angles. This value was comparable to those for 101, suggesting that the frequency characteristics of the linear component are similar to those in 101. On the other hand, second-order kernels from 102 and 103 were very similar to each other (Fig. 6). The kernels had an elongated depolarizing peak on the diagonal line where $\tau_{1}=\tau_{2}$ with two off-diagonal valleys at $\tau_{1} \neq \tau_{2}$. This configuration was basically invariant irrespective of the stimulus angle. The magnitude of the valleys and peaks, however, were reduced when the stimulus angles were ipsi $30^{\circ}$ and cont $150^{\circ}$, which was accompanied by a significant decrease in amplitude of the first-order kernel.

Figure 7 shows responses from 102 evoked by white noise at ipsi $150^{\circ}$ and ipsi $60^{\circ}$, and the corresponding linear and secondorder models. When stimulated at ipsi $150^{\circ}$, the summation (labeled Sum) of the two models accurately predicted the cell's actual response with an MSE of $37.7 \%$ (Fig. $7 A_{1}$ ). In this recording, MSE for the linear model was $76.3 \%$. There was therefore a $38.6 \%$ improvement in the MSE by adding the second-order model. The power spectrum of the response and second-order model began to fall off at $20-30 \mathrm{~Hz}$, whereas that of the first-order model had a peak at 70-90 $\mathrm{Hz}$ (Fig. $7 B_{t}$ ). Both of the linear and second-order nonlinear components were very small for the ipsi $60^{\circ}$ wind stimulation, where MSEs for the models were $93.9 \%$ and $80.6 \%$, respectively (Fig. $7 A_{2}$ ). Indeed, they had a power much lower than that of the response (Fig. $7 B_{2}$ ).

\section{Interpretation of the nonlinearity in 102 and 103}

Figure 8 shows two examples of the second-order kernel from 102 at cont $30^{\circ}$ and ipsi $150^{\circ}$. The kernels had an elongated, depolarizing peak on the diagonal line (Fig. $8 A$ ). The kernels generated by 102 and 103 showed this pattern irrespective of stimulus angle. This was because the second-order kernels were quadratic for input.

In Figure $8 B$, the first-order kernel (solid line) computed from the same recording as shown in Figure $8 A$, on which the diagonal cut, the side view of the second-order kernel, is superposed (broken line). The first-order kernel is biphasic, whereas the diagonal cut is monophasic. At either stimulus angle, the hyperpolarization of the first-order kernel was offset by the depolarizing peak of the second-order kernel. On the other hand, the depolarization of the first-order kernel is enhanced by the diagonal cut. This is a compression nonlinearity, and implies that the interneuron responds by producing a depolarizing potential only when wind is directed from the front. This was confirmed by model predictions for 102, as shown in Figure $8 C$. In this recording (stimulated at ipsi $150^{\circ}$ ), the summation of the linear and second-order models showed a good match with the actual response with an MSE of $38 \%$ for the second-order model. We note that a hyperpolarization in the linear model was compressed by a depolarization of the second-order model (arrows in Fig. 8C). 
1st-order kerne

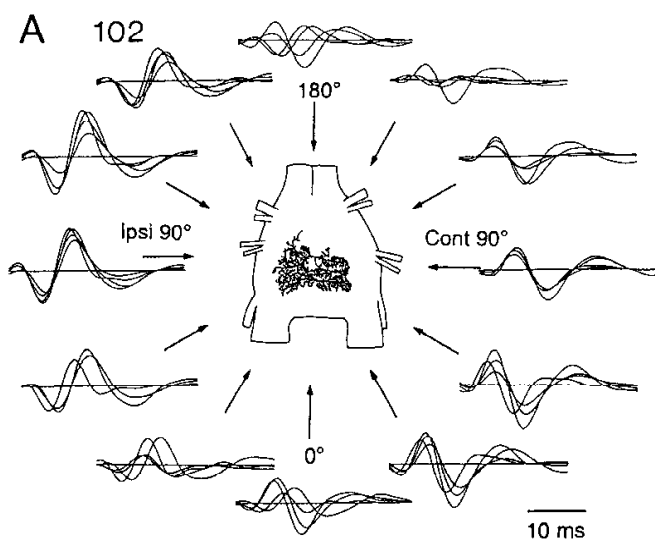

Figure 6. First-and second-order kernels in 102 and 103 at different stimulus angles. All kernels were normalized. In left diagrams, four first-order kernels from different animals were superposed. Note that the waveform of the kernel was reversed at ipsi $60^{\circ}$ and cont $120^{\circ}$ in 103 , and ipsi $30^{\circ}$ and cont $150^{\circ}$ in 102. When stimulated anteriorly, the interneurons produced the first-order kernel with an initial hyperpolarization. The kernel was, however, initially depolarized when stimulated posteriorly. The second-order kernels shown in the right diagrams here were closely similar to each other, and had a diagonal depolarizing peak with two off-diagonal hyperpolarizing valleys. Unlike the first-order kernels, no directional sensitivity was found in the signature of the second-order kernel.

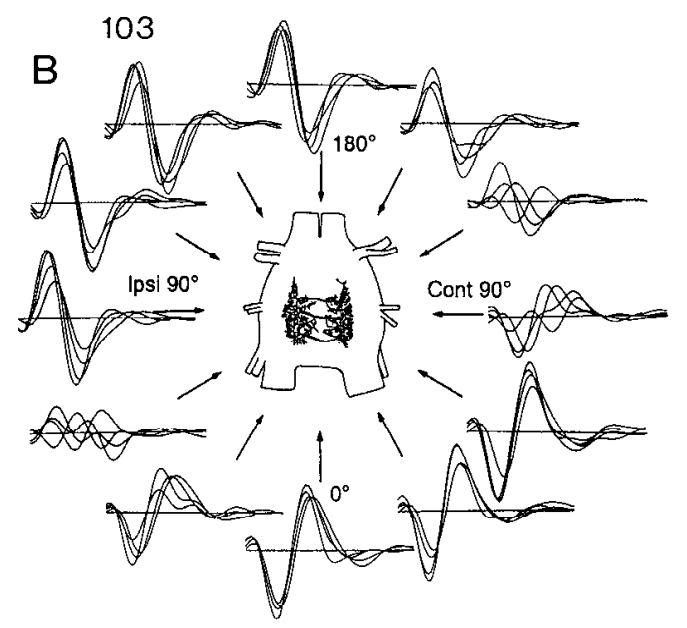

2nd-order kernel
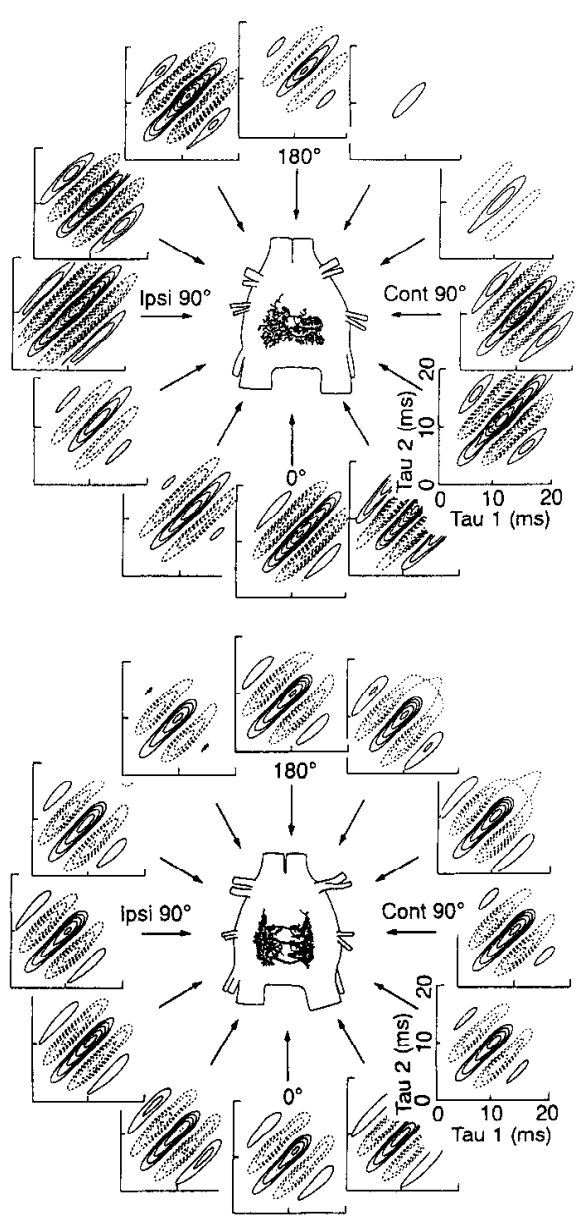

Interneurons 104, 108, and 201

The third type comprises interneurons 104,108 , and 201, whose neuronal structures are shown in Figure 9. The cell body of 104 is approximately $35 \mu \mathrm{m}$ in diameter and lies in the ventral cortex of the ganglion. The primary neurite arising from the cell body expands abruptly in diameter over $30 \mu \mathrm{m}$ and gives off numerous fine processes posteriorly. After crossing the midline, the primary neurite turns posteriorly and then branches repeatedly to form the field of densely packed branches. Interneuron 201 has a cell body with a diameter of approximately $20 \mu \mathrm{m}$ near the base of the cercal nerve. The primary neurite bifurcates to extend two main neurites anteriorly and posteriorly. The anteriorly directed neurite runs parallel to the midline for 700 $800 \mu \mathrm{m}$ to terminate, whereas the posteriorly directed neurite subsequently turns anteriorly and ventrally to run along the ventral edge of the neuropil, forming a denscly packed dendritic field. Interneuron 108 has a cell body with a diameter of 20-30 $\mu \mathrm{m}$ lying laterally in the dorsal cortex of the ganglion. The primary neurite runs medially before turning sharply in a ventrolateral direction at a point $100 \mu \mathrm{m}$ from the midline, and then bifurcates in a T-shape to send two major neurites anteriorly and posteriorly. They are further subdivided into two thick neurites that send out numerous branches on both sides of the ganglion.

Figure 10 shows first- and second-order kernels produced by stimuli delivered at various angles for 104, 108, and 201. We note that the first-order kernels were very small or noisy in any stimulus direction. The second-order kernels did not show any directional preference; the 12 kernels were very similar in their signature as well as in their magnitude. The kernels in 104 had an elongated hyperpolarizing valley on the diagonal line (Fig. $10 \mathrm{~A}$ ), whereas those in 108 and 201 had a depolarizing peak (Fig. 10B,C). Both kernels also had two off-diagonal valleys in 201 and 108, and two off-diagonal peaks in 104. Thus, this kernel pattern closely resembles that seen in 102 and 103 .

Figure 11 shows a typical example of responses in 104, which was evoked by white noise and sinusoidal of wind delivered along the long axis of the animal's body. The wind stimulus usually produced a hyperpolarization in 104 . The second-order model matched well with the actual response, whereas the firstorder (linear) model was very small (Fig. 11A). Indeed, the MSE for the linear model was $98.9 \%$, whereas that for the secondorder models was $42.7 \%$. Thus, there was $56.2 \%$ improvement of MSE after adding the second-order component. Figure $11 \mathrm{C}$ shows the power spectra of the input white noise and responses at two different angles (ipsi $90^{\circ}$ and $0^{\circ}$ ). The second-order nonlinear component had a power comparable to that of the response at $0^{\circ}$, whereas that of the linear component was much smaller (Fig. $11 C_{1}$ ). A similar observation was made at the stimulus angle of ipsi $90^{\circ}$ (Fig. $11 C_{2}$ ), confirming our observation that the response in 104 was omnidirectional. In Figure $11 B$, the linear and second-order models were also predicted 
A1 Interneuron 102

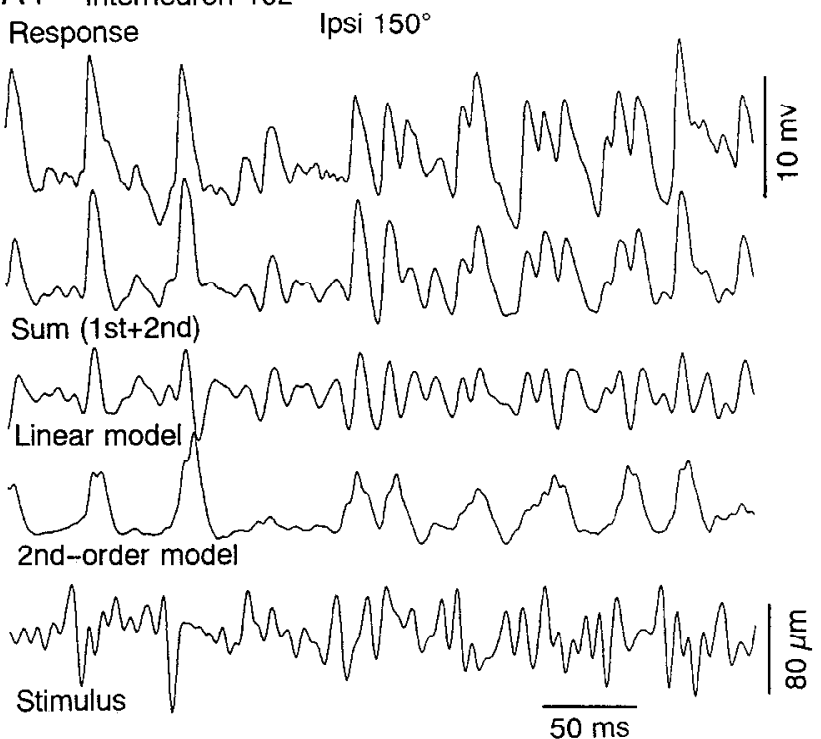

B1

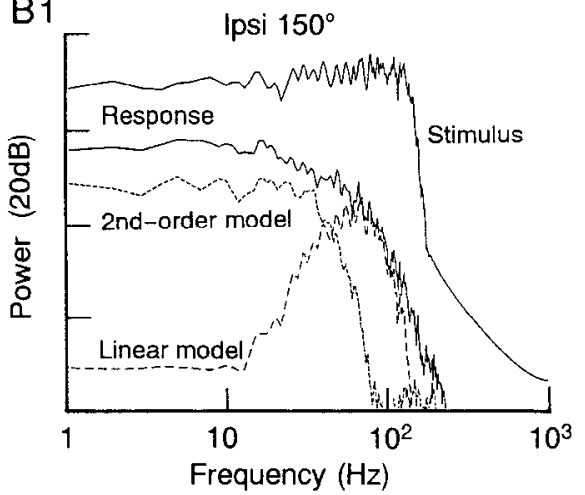

A2

\section{Response}

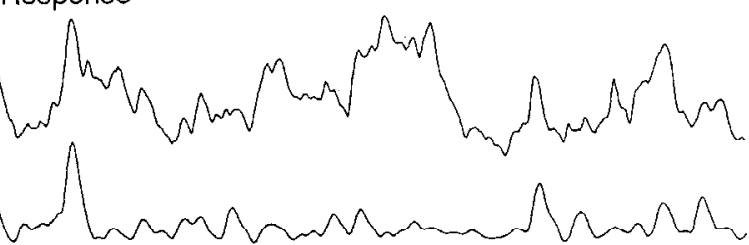

Sum (1st+2nd)

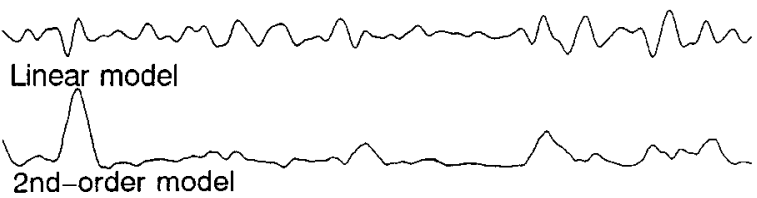

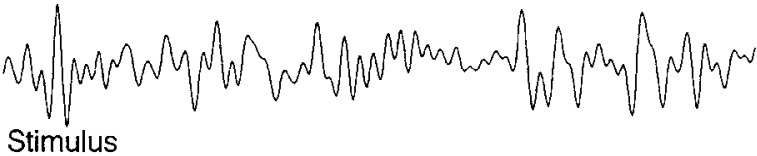

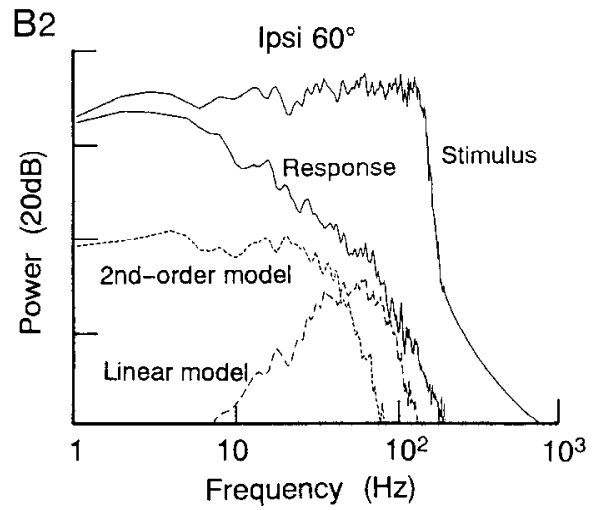

Figure 7. $A$, Intracellular recordings from 102 to white noise at ipsi $150^{\circ}\left(A_{f}\right)$ and ipsi $60^{\circ}\left(A_{2}\right)$, and the corresponding first- and second-order models. At ipsi $150^{\circ}$, the summation of the first- and second-order model predicted the cell's actual response very well. The MSEs for these two models were $76.3 \%$ and $37.7 \%$ at ipsi $150^{\circ}$, and $93.9 \%$ and $80.6 \%$ at ipsi $60^{\circ}$, respectively. The summation trace $(S u m)$ was produced by summing the linear and second-order nonlinear model traces. $B$, Frequency responses in 102, showing power spectra of the responses; kernel predicted models are shown for $150^{\circ}\left(B_{1}\right)$ and ipsi $60^{\circ}\left(B_{2}\right)$ stimulus.

by convolutions of the input sinusoidal sweep with the secondorder kernels. The second-order model as well as the actual response had a hyperpolarizing trend at 70-100 Hz. Although the power spectrum for the response showed a low-pass filter nature, the interneuron produced no significant response at a lower frequency range. We also note that the power spectrum of the response is beyond the frequency range of the stimulus. This is consistent with our observation that 104 shows a frequency-doubling response to a sinusoidal wave (Fig. $11 B$; see also Fig. $12 C$ ). The electrical stimulation of the cercal nerves on the ipsilateral side evoked a hyperpolarization that was 8$10 \mathrm{mV}$ in amplitude with a latency of $2.5 \mathrm{msec}$ (Fig. $11 \mathrm{D}$ ). The stimulation on the contralateral side elicited a small (2-3 mV) depolarization with a latency of $1.5 \mathrm{msec}$, which was followed by a hyperpolarization.

Responses in 108 and 201 were very similar to each other, and were reasonably well predicted by the second-order kernel. Figure $12 \mathrm{~A}$ shows a typical example of the response in 201, which was evoked by white noise modulation of wind. MSEs for the linear and second-order nonlinear models were $99.0 \%$ and $42.4 \%$, respectively. Frequency characteristics of the response evoked in 108 and 201 were nearly the same as in 104
(Fig. 12B). The power spectra of the white noise-evoked response had their cutoff frequency at $20 \mathrm{~Hz}$ (Fig. 12B $B_{l}$ ). The second-order model had power almost comparable to that of the response (Fig. 12 $B_{2}, B_{3}$ ), whereas the linear model had a power of $20-40 \mathrm{~dB}$ less than the actual response, indicating that the linear component comprises only a minor part of the response. In this type of interneuron, a frequency-doubling response was substantial when stimulated by a sinusoidal wave at a higher-frequency range (Fig. 12C). This was expected from the fact that the wind-evoked responses in 104 and 201 were best approximated by the second-order kernel, a squaring function.

\section{Modeling of the second-order nonlinearity}

Figure 13 shows a diagram of the generation of the second-order kernel in a 201 interneuron, which has a characteristic threebar structure with an elongated depolarizing peak on the diagonal and two off-diagonal hyperpolarizing valleys. The second-order nonlinearity was reproduced by a filter cascade of Korenberg type (Korenberg and Hanter, 1986). The arrangement was a linear (band-pass) filter followed by a static nonlinearity (square) and a second linear (low-pass) filter (Fig. 13A). White noise input 

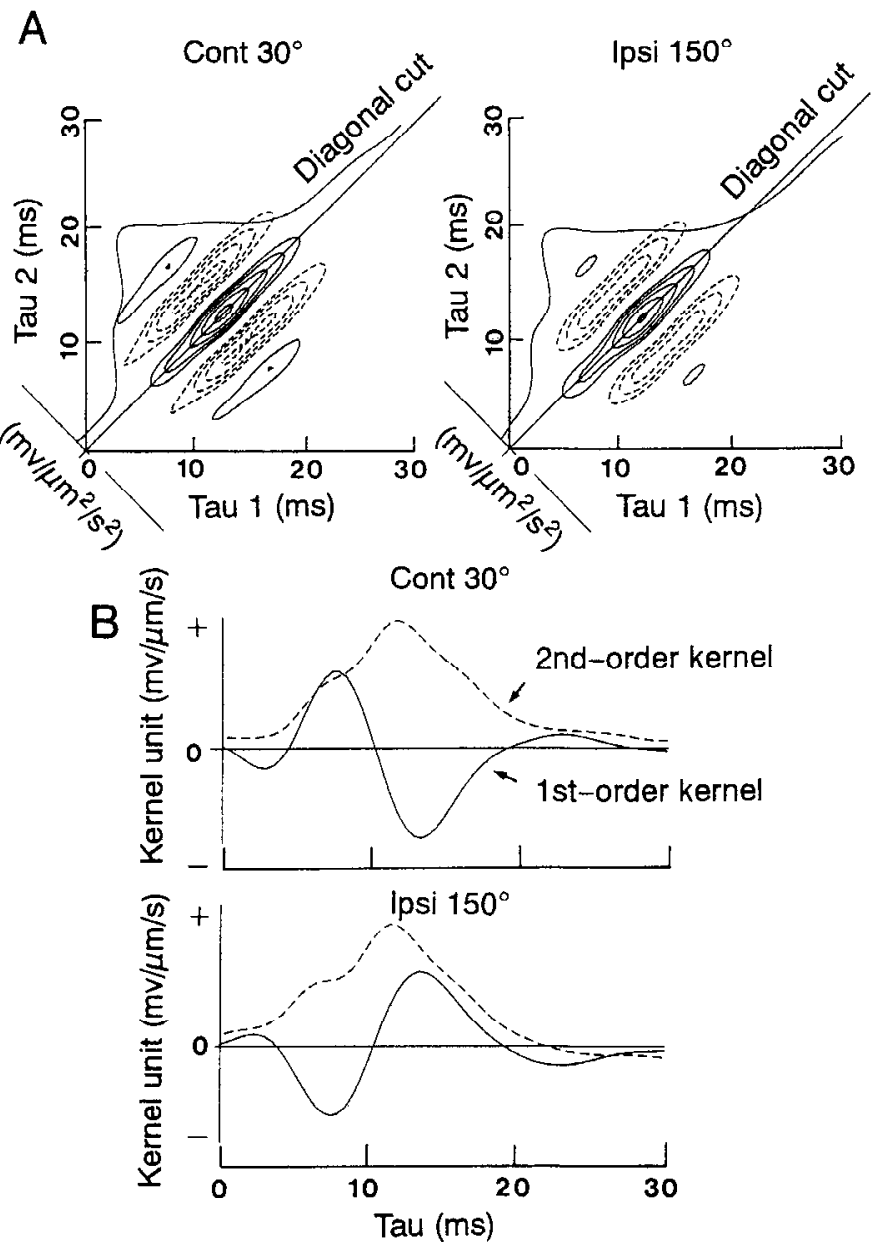

C

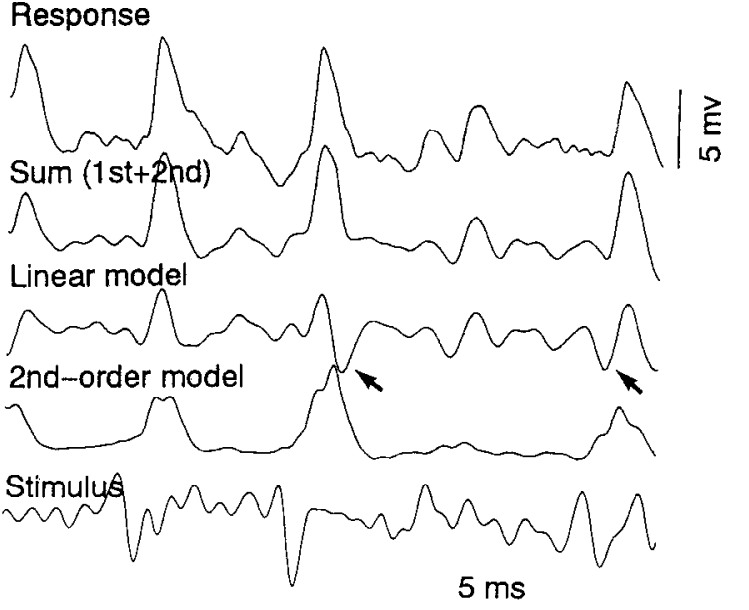

Figure 8. A, Contour plots of second-order kernels from 102 when stimulated at cont $30^{\circ}$ and ipsi $150^{\circ}$, on which the diagonal cuts of the kernels were superimposed. The kernel had an elongated depolarization peak on the diagonal with two off-diagonal valleys. $B$, The diagonal cuts at the second-order kernels at $180^{\circ}(l e f t)$ and $0^{\circ}$ (right) are shown by the broken lines. The figure also shows the first-order kernels from the same cell (solid lines) and the summation traces (broken lines), which were produced by summing the trace of the first-order kernel and that of the diagonal cut. Note that the hyperpolarizing peak of the first-order kernel was offset by the depolarizing peak of the second-order kernel; this is a compression nonlinearity. $C, \mathrm{~A}$ part of the record, the response trace and its linear and second-order models in Figure $7 A_{l}$, is expanded. Note that a hyperpolarization of the first-order model, indicated by arrow, was opposed by a depolarization of the second-order model. The summation traces suggest that the interneuron produces a depolarizing potential only when a wind is directed from the head to tail. was initially passed through a band-pass filter. In this case, the input was convolved with a parallel cut of the second-order kernel that was used as a band-pass filter (Fig. 13B). The bandpass filter's output was squared, and then passed through a lowpass linear filter (Fig. 13D). The cascade model was then crosscorrelated against the original input to compute a second-order kernel. The two kernels, one from 201 (Fig. 13B) and the other synthesized (Fig. $13 C$ ), are very similar, consisting of an elongated depolarizing peak with two off-diagonal valleys. The squared output is therefore a reproduction of 201's response. Indeed, the second-order model to a sinusoidal wave had a second harmonic at high-frequency range.

\section{Directional response curves}

MSE for a kernel prediction represents the ratio of the deviation against the actual response, and thereby evaluates the accuracy of the kernel prediction. Thus, plots for MSEs on the polar ordinate allow us to draw directional response curves for the linear and nonlinear part of the response, as illustrated in Figure 14. In $101,107,111$, and 203, the linear component is directionally sensitive, and comprises about $60 \%$ of the total response at least for 101,107 , and 111 in preferred directions (i.e., the first-order model had an MSE of less than 40\%) (Fig. 14A). It forms two response ranges that are symmetrically arranged across the null directions $\left(0^{\circ}\right.$ and $180^{\circ}$ for 101 and 107 , and ipsi $60^{\circ}$ and cont $120^{\circ}$ for 111 and 203). On the other hand, the secondorder component contributes only a few percent to improve MSE regardless of the stimulus angle. The plots from 102 and 103 show that both first- and second-order components are directionally sensitive, and each comprises $20-30 \%$ of the total response in a preferred direction in those interneurons (Fig. $14 B$ ). Moreover, the first-order component has two response ranges approximately quadrant, which are symmetrically arranged across null directions, ipsi $60^{\circ}$ and cont $150^{\circ}$. The directional curves in 104, 108, and 201 show that responses in these interneurons are omnidirectional, and that the secondorder component comprises a major part $(50-60 \%)$ of a total response (Fig. 14C). The linear component is minor, as suggested by MSEs for the first-order model, which were $90-95 \%$ on average.

\section{Discussion}

Three sets of nonspiking local interneurons are recognized on the basis of the kernel analysis in the terminal ganglion of the cockroach. The first group, in which the response dynamics are characterized mainly by differentiating first-order kernels, includes interneurons $101,107,111$, and 203. The second, in which the wind evoked response is best approximated by the summation of the first- (linear) and second-order models, includes interneurons 102 and 103, while the third, in which the response is best approximated by the second-order model, includes interneurons 104, 201, and 108. Thus, response to wind in the first group is linear, whereas those in the latter two groups are nonlinear. Some of them have dendritic arborizations on both sides of the ganglion, and others only on one side. No morphological correlation to the physiological classification of interneurons has been revealed. For example, 203 is physiologically of linear type whereas 201 is of nonlinear type, although these two interneurons occupy similar dendritic fields within the ganglion.

In insect segmental ganglia, spiking local interneurons are largely responsible for the local processing of primary sensory 


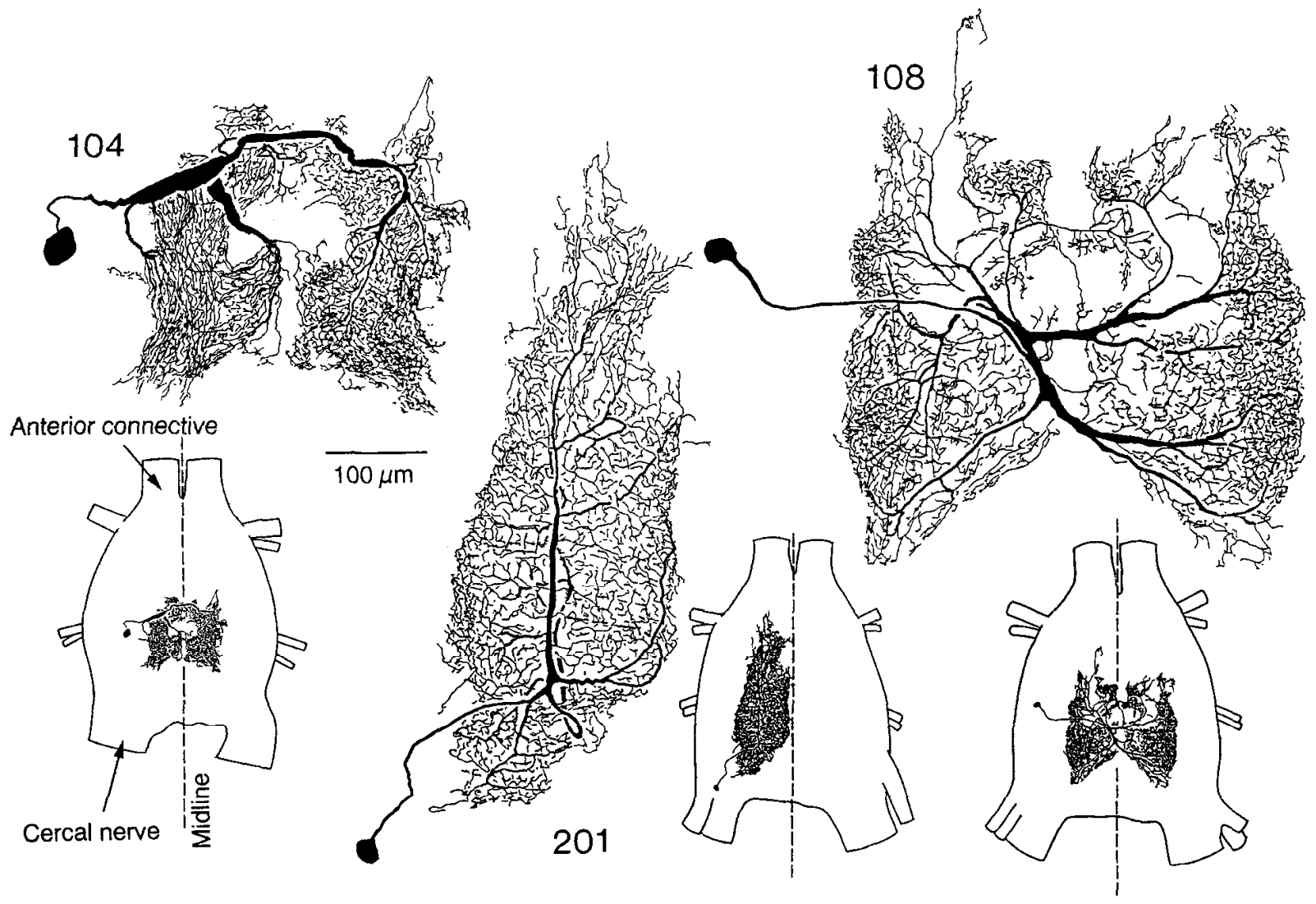

Figure 9. Morphology of the other type of nonlinear interneurons, viewed dorsally. Broken line indicates the midline of the ganglion.

inputs whereas nonspiking ones play a major role in the control and coordination of motor output (Siegler, 1984). For example, spiking local interneurons in the metathoracic ganglion of the locust receive inputs directly from afferents of hairs and campaniform sensilla in a way such that a particular region on the surface of a hind leg is mapped onto these interneurons (Burrows and Siegler, 1985). They make direct inhibitory connections onto premotor, nonspiking local interneurons on which intraand intersegmental input converges (Siegler and Burrows, 1983; Burrows, 1987). Nonspiking local interneurons control the pools of motor neurons required for the different patterns of limb movement (Burrows, 1980). However, in the cockroach, we showed that sensory input from cerci is processed by many nonspiking local interneurons, as it is in the cricket (Kobashi and Yamaguchi, 1984; Bodnar et al., 1991) and the locust (Boyan et al., 1989).

\section{Code for the stimulus direction}

Figure 15 summarizes the results showing the organization of wind receptive fields of the nine nonspiking local interneurons. Polar plots of MSE for the models revealed bilobed directional response curves for $101,107,111$, and 203. The first-order kernel in a given direction was the mirror image of that in the opposite polar direction. The polarity reversal occurred along the midline of the animal for 101 and 107, and roughly orthogonal to the midline for 111 and 203. Thus, 101 and 107 must be excited on the ipsilateral side and inhibited on the contralateral side, while 111 and 203 must be excited by a wind roughly from the rear and inhibited from the front. Cells 102 and 103 are also directionally sensitive, being excited in one direction but receiving no or weak inhibition in the other direction (as suggested in Fig. 8): 102 is excited by a wind from the rear, and 103, by wind from the front. On the other hand, interneurons 104, 108, and 201 are omnidirectional, being excited or inhibited in all directions. The pattern of directional sensitivity in some of these interneurons are closely similar to those of some GIs (Westin et al., 1977). For example, GIs 1 and 2 are omnidirectional, as are 108 and 201, being excited in all directions, while GI 3 responds to wind from in front of the animal, just as does 103 .

The selective projections of those hairs onto these interneurons would account for their directional sensitivity. Almost 220 wind receptive hairs (filiform sensilla) on an adult cercus are organized spatially into columns and rows. Each of the 19 cercal segments of the adult has a row of nine hairs, except for the segments near the tip and base, which range from 0.5 to $1 \mathrm{~mm}$ in length (Nicklaus, 1965). All hairs of a given column have the same optimal wind directions that differ from column to column (Dagan and Camhi, 1979). Daley and Camhi (1988) showed that GIs 1 and 2 receive excitatory inputs from the same set of ipsilateral sensory neurons, that is, those of all nine scnsory columns, while GI 3 receives excitatory input only from ipsilateral columns $d, f, g, i$, and $k$, all of which have their optimal excitatory directions within the boundaries of the response curve of GI 3.

The cockroach responds to wind stimulus with an escape that begins with a rapid directional turn, and runs away from its predator (Camhi and Tom, 1978; Camhi, 1980). Seven pairs of 

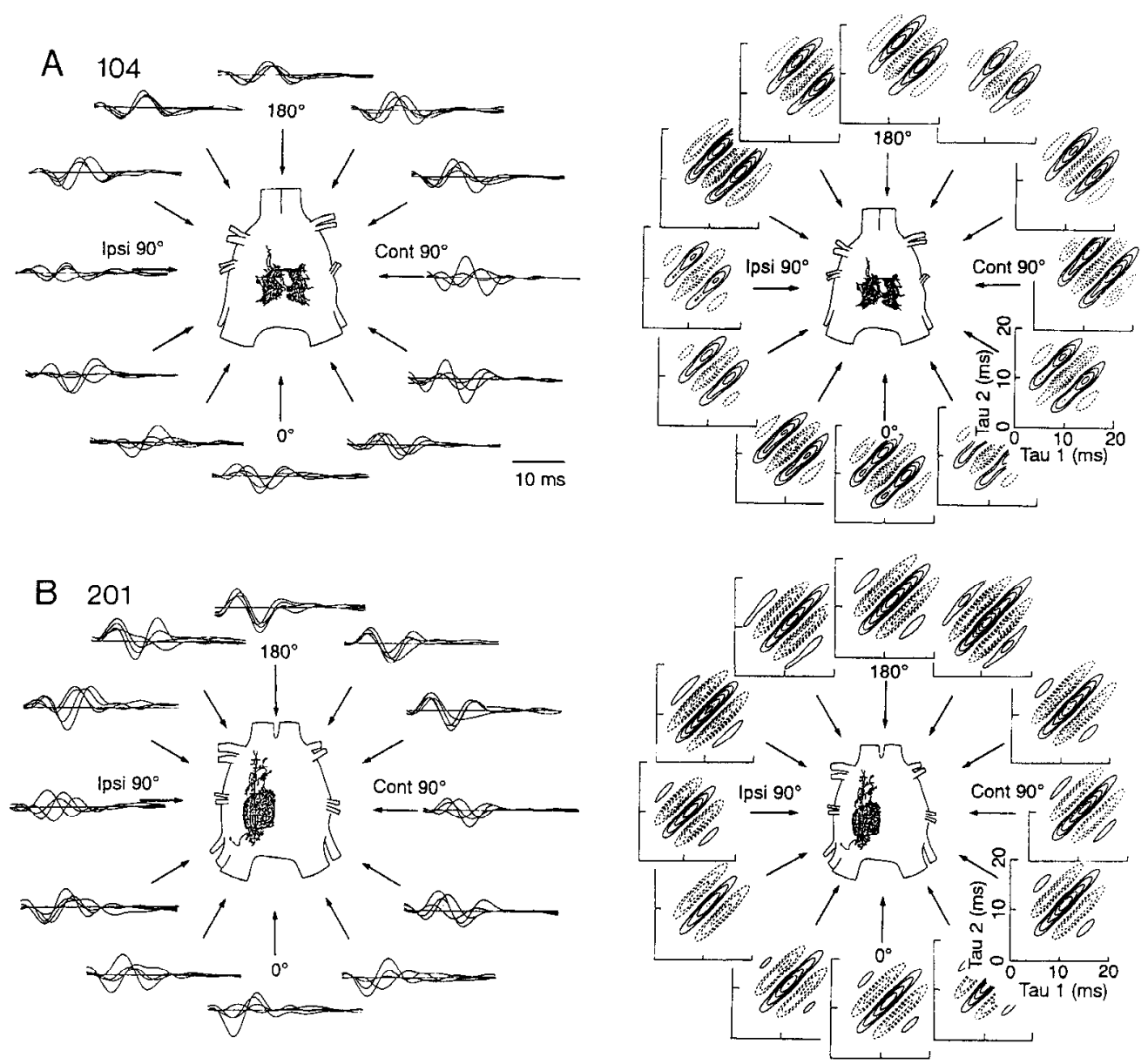

Figure 10. Polar representations of first- and second-order kernels in 104, 108, and 201. All kernels were normalized. In left column, four kernels from four different animals were superimposed at each stimulus angle in
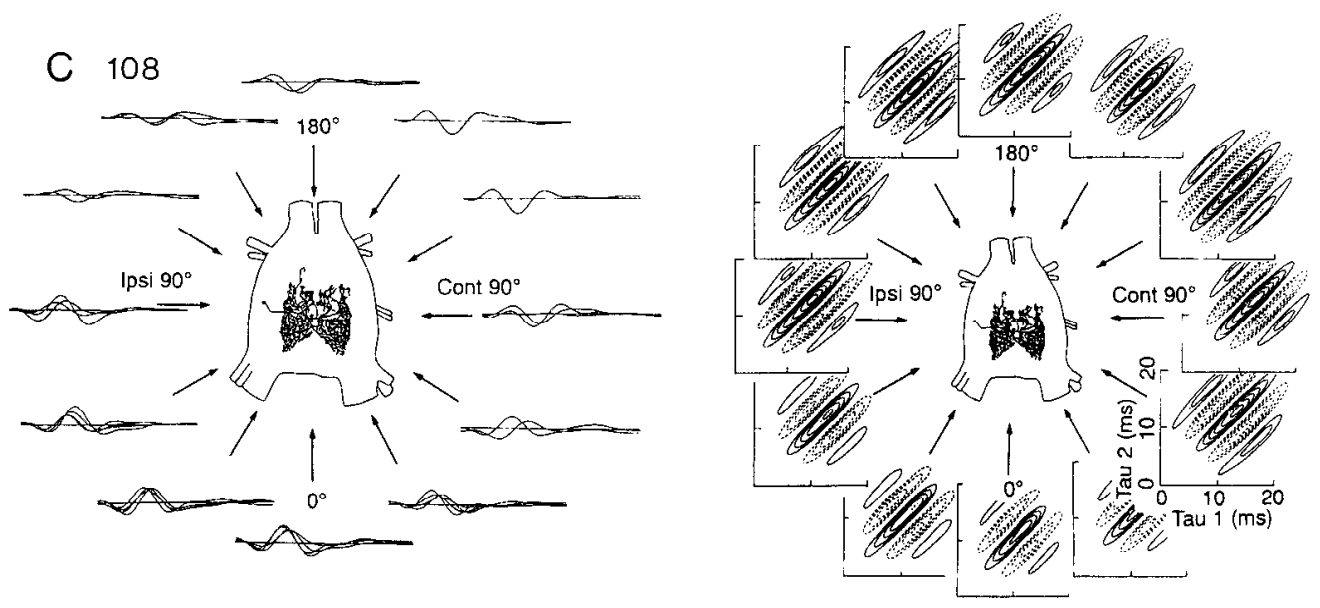
104 and 201, and three kernels in 108.

GIs have been suggested to elicit this directional escape response: electrical stimulation of individual GIs elicits excitation of leg motor neurons associated with escape (Ritzmann and Camhi, 1978; Ritzmann, 1981; Ritzmann and Pollack, 1981), and killing of the GIs can alter wind-evoked motor responses (Westin and Ritzmann, 1982). Of these, the ventral four GIs (GIs 1-4) are especially important in evoking the escape behavior under most natural conditions (Camhi and Levy, 1989). However, none of the GIs respond selectively to wind from the left versus right. For example, GIs 1, 2, and 4 are all omnidi- rectional, whereas GI 3 responds to wind from the front (Westin et al., 1977). Therefore, Camhi and Levy (1989) proposed a model of the assembly code for direction that accounts for this discrepancy, in which the number of action potentials is compared between the left versus the right group of ventral GIs (GIs 1-4). Our findings of the directionally selective interneurons provide new insights into the neural mechanism responsible for the specification of stimulus direction. We observe that 101 and 107 respond most preferentially to wind from the left versus right, whereas $102,103,111$, and 203 respond to that from the 

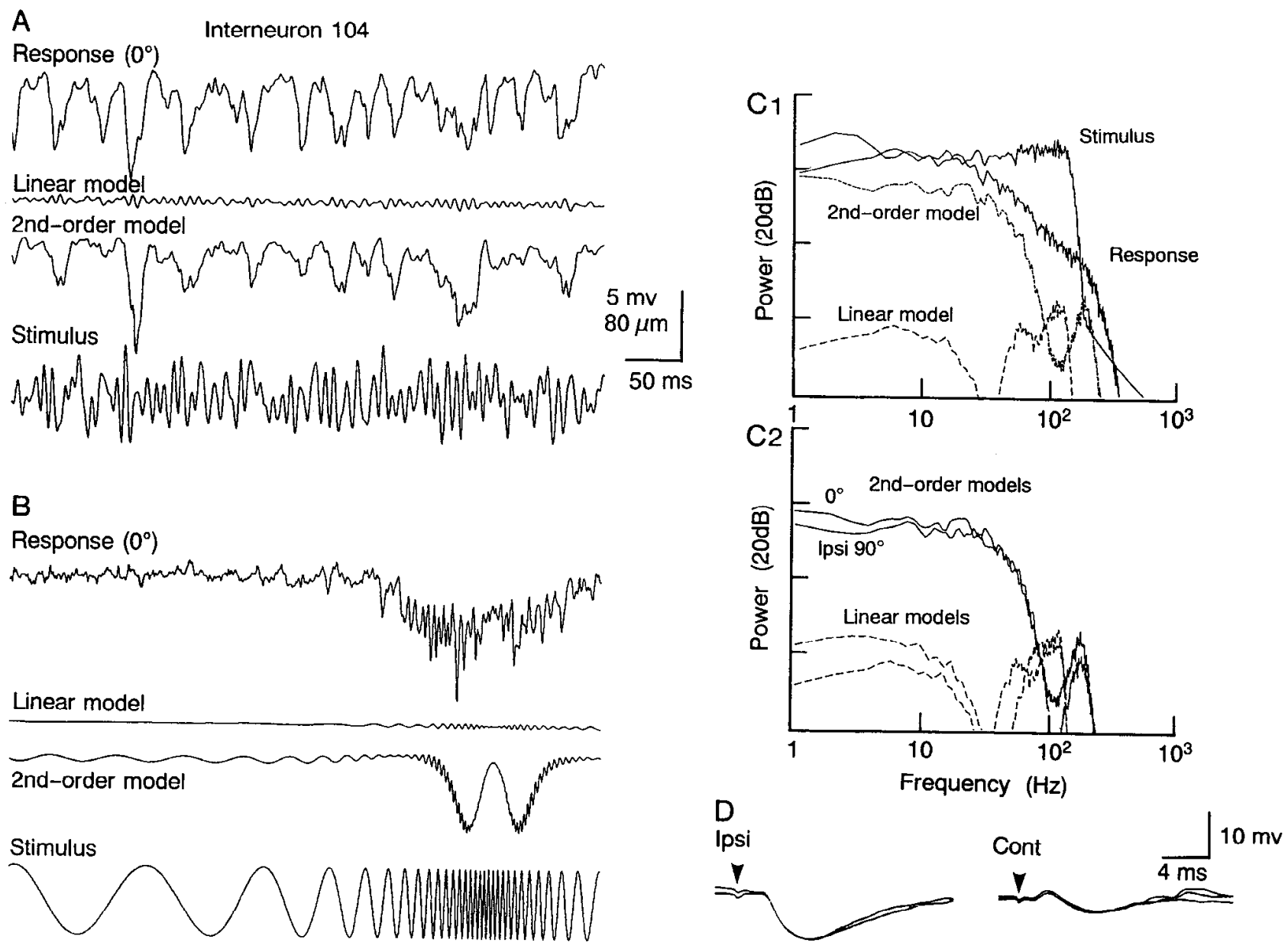

Figure 11. A, Response to white noise modulation of wind in 104, and its linear and second-order nonlinear models. MSEs for the linear and second-order nonlinear models were $98.9 \%$ and $42.7 \%$, respectively. The improvement of MSE by the second-order model was $56.2 \%$ in this record. The second-order nonlinear model matches the actual response well. $B$, Hyperpolarized response from the 104 evoked by the sinusoidal modulation of wind at $0^{\circ} . C$, Power spectra of the responses $\left(C_{1}\right)$ and linear (first-order) and second-order models $\left(C_{2}\right)$ at two different stimulus angles $\left(0^{\circ}\right.$ and ipsi $90^{\circ}$ ). We note that the response exhibited power in a frequency range beyond that of the stimulus itself. $D$, Responses from a 104 interneuron evoked by electrical stimulation (arrowheads) of the ipsilateral and contralateral cercal nerves. Three sweeps were individually superimposed.

front versus rear (Fig. 15). There are thus at least two subsystems responding maximally to wind displacement along two coordinate dircctions, one for the longitudinal direction and the other for the transverse direction, into which the direction (and intensity) of wind is encoded. For each direction, each system is tuned to respond to one of the two vector components of this direction. A similar neural coding is seen in the fly visual system, where the direction and velocity of a motion pattern on the retinal image is encoded into two independent motion-detecting circuits, a vertical system (VS) and a horizontal system (HS). The VS and HS neurons in the lobula plate respond maximally to vertical and horizontal motion, respectively (Hausen, 1981), and are thought to play a major role in the control of optomotor behavior (Hausen and Wehrhahn, 1983; Egelhaaf et al., 1988).

Another important aspect of the function of directionally sensitive interneurons is "lateral inhibition." A population of GIs in the terminal ganglion of the cricket responds to wind with sharply directional sensitivity owing to converging excitatory and inhibitory input in their receptive field (Matsumoto and Murphey, 1977; Jacobs et al., 1986). The inhibitory input serves to enhance the contrast between two cerci (lateral inhibition), and thereby to sharpen the directional sensitivity of the GIs, although no such interneurons have yet been described. In other integrative systems, some interneurons mediate lateral inhibition that cnhances the contrast of stimulus from left versus right. For example, omega cells in cricket thoracic ganglia inhibit their contralateral counterpart (Wohlers and Huber, 1978), and local, directionally selective interneurons in crayfish terminal ganglion inhibit the intersegmental interneuron on the contralateral side (Reichert et al., 1983; Kondoh and Hisada, 1986). Cells 101 and 107 are similar to those interneurons in some respects, and thus are the most plausible candidate for lateral inhibition between two cerci. First, they all are local interneurons, and responsible for local circuit interactions within the segmental ganglion. Second, they are selectively excited by a stimulus with a particular directionality, and inhibited in the other direction (for the LDS interneuron, Krenz and Reichert, 1985). Third, they have extensive bilateral dendritic arborizations that are interconnected by a thick neurite running across the midline.

\section{Origin of the differentiating first-order kernel}

When applied to a linear system, the first-order kernel corresponds to the system's impulse response. When applied to a nonlinear system, the kernel is the best (in an MSE sense) linear 

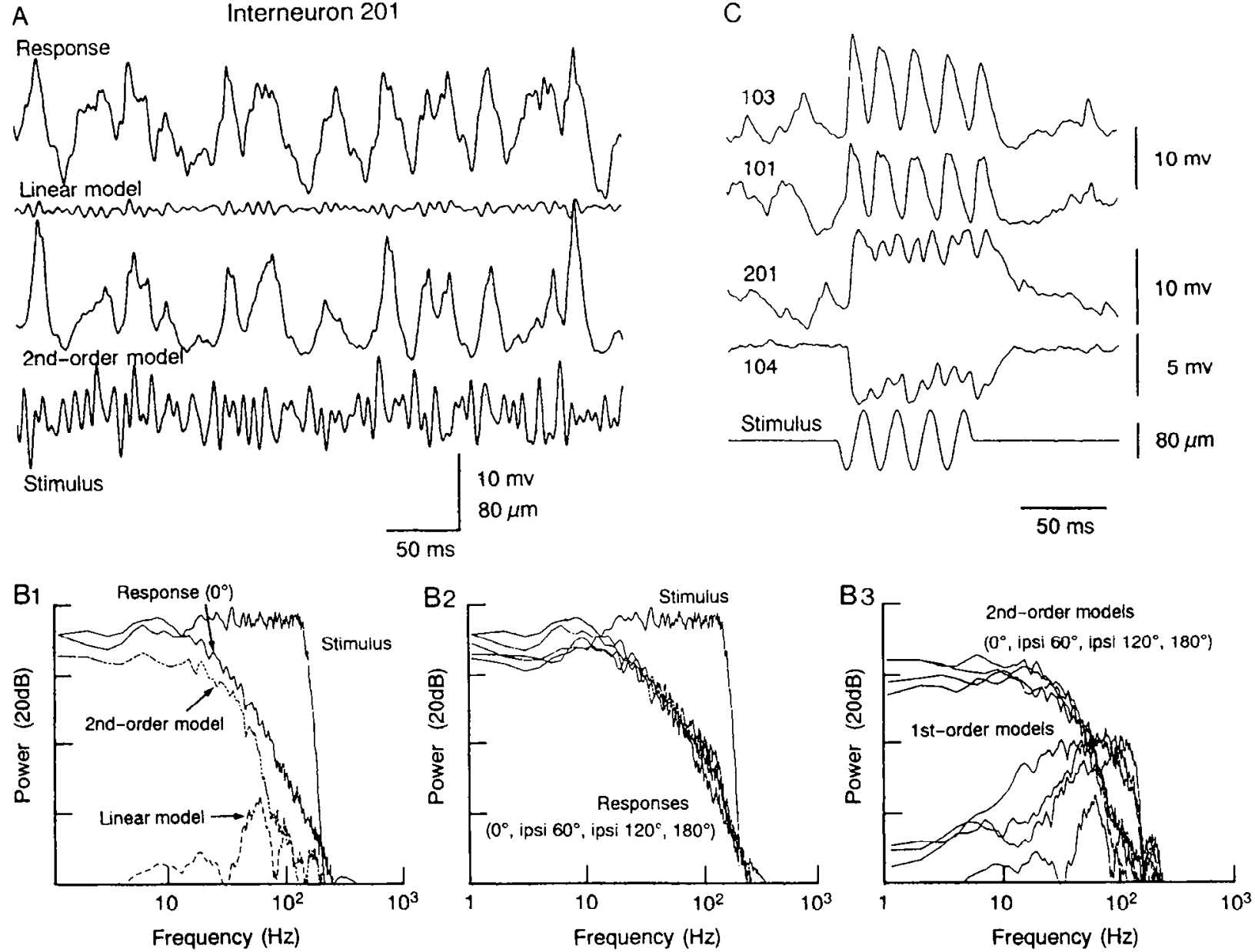

Figure 12. A, Intracellular recordings from 201 to white noise stimulus, and its linear and second-order nonlinear models. MSEs for the linear and second-order nonlinear models were $99.0 \%$ and $42.4 \%$ in this recording. Therefore, there is a $56.6 \%$ improvement of the MSE with inclusion of the second-order model. $B_{1}$, Frequency response in 201 showing power spectra of the input white noise, response, and linear and second-order models when the interneuron was stimulated at $0^{\circ}$. We note that the synaptic input onto 201 and the second-order model is passed through a lowpass filter. $B$, and $B$, Power spectra of responses, linear models, and second-order nonlinear models in four different directions $\left(0^{\circ}\right.$, ipsi $60^{\circ}$, ipsi $120^{\circ}$, and $180^{\circ}$ ). $C$, Intracellular recordings from $101,103,104$, and 201 to a sinusoidal stimulus, in the best directions for cells 101 and 103 , showing a frequency-doubling response in 104 and 201 . In contrast to the linear $(101)$ and half-wave rectifier (103) interneurons, responses to a sinusoidal wave at a high-frequency range in 104 and 201 had a second harmonic component.

approximation of the system's impulse response. For example, in horizontal cells in a turtle retina, the first-order kernel is equivalent to the response to a brief flash of light (Chappell et al., 1985; Sakai and Naka, 1987). In the cockroach, the firstorder kernel is the best linear approximation of the cell's response to a brief push-pull of the speaker's moving plate and hence a brief air displacement. The waveform of the first-order kernels from 101,107 , and 203 was biphasic, whereas that from 111 was more differentiating, indicating that these interneurons are sensitive to the rate of change of the input (i.e., wind velocity). The wind receptors are also velocity sensitive (Buño, 1981; Kondoh et al., 1991b). The initial excitation and the following inhibition of the biphasic first-order kernel corresponds to a response to wind from a given direction and the opposite polar direction, respectively. If the receptors respond linearly to the bending of sensory hair and the air displacement, the results described here can be explained. However, the cercal afferents respond in a nonlinear way such that bending of a hair to the direction opposite to the optimal excitatory field produces no response in its receptor neuron or inhibits its spontaneous activity (Dagan and Camhi, 1979).
A likely possibility is that the first-order kernel produced by the contralateral stimulation is transmitted to the interneurons via a sign-inverting synapse, and two subsets of column hairs whose optimal directions are opposite to each other act in a push-pull fashion. For example, 101 would receive excitatory input directly from the filiform hairs of columns $\mathrm{I}$ and/or $\mathrm{m}$, and inhibitory input from columns a and/or $d$ via an unknown inhibitory interneuron intervening between them. Electrical stimulation of the cercal nerve revealed that 101 receives postsynaptic, inhibitory input from cercus in addition to short-latency, probably monosynaptic excitatory inputs (Kondoh et al., 1991a). Postsynaptic inhibition has been previously demonstrated in the cercal system of cockroaches (Callec, 1974) and crickets (Levine and Murphey, 1980). Our unpublished observation on changes induced in the cockroach after application of picrotoxin shows that inhibitory interaction plays an important role in linear processing of the signal by linear interneurons. Picrotoxin is well known to act as a noncompetitive antagonist of GABA. As we expected, MSE for the first-order model significantly increased $4 \mathrm{~min}$ after being bathed in $10^{-4} \mathrm{M}$ picrotoxin, indicating that the polarity-inverted kernels are produced 
A

$L-N-L$ cascade model

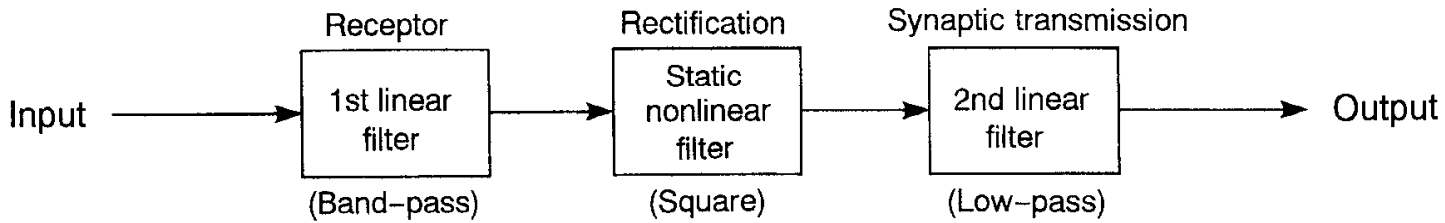

B

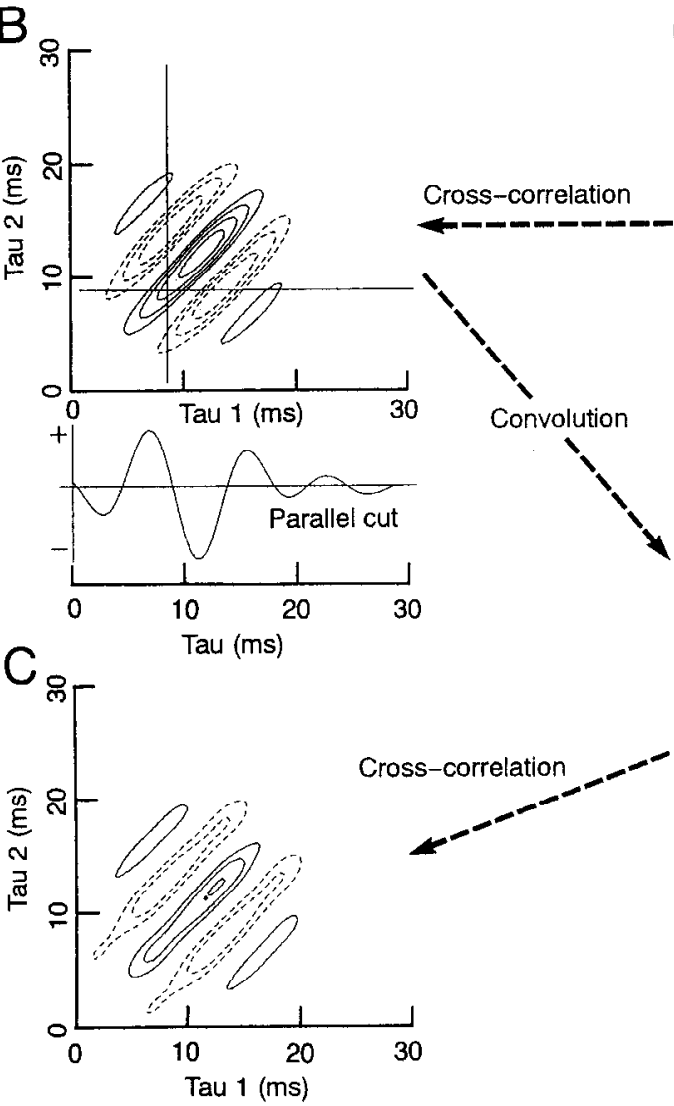

D

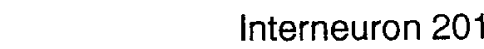




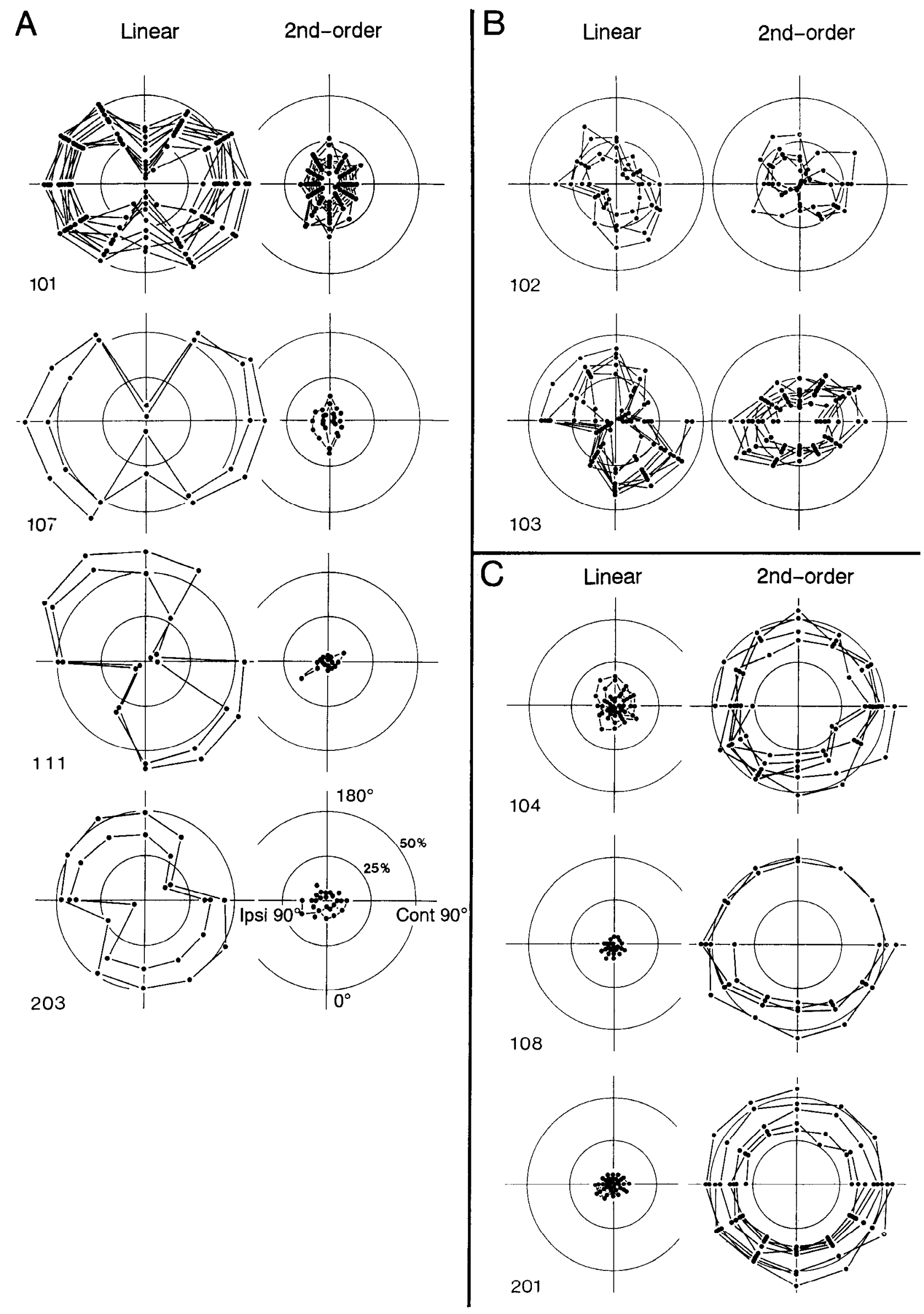



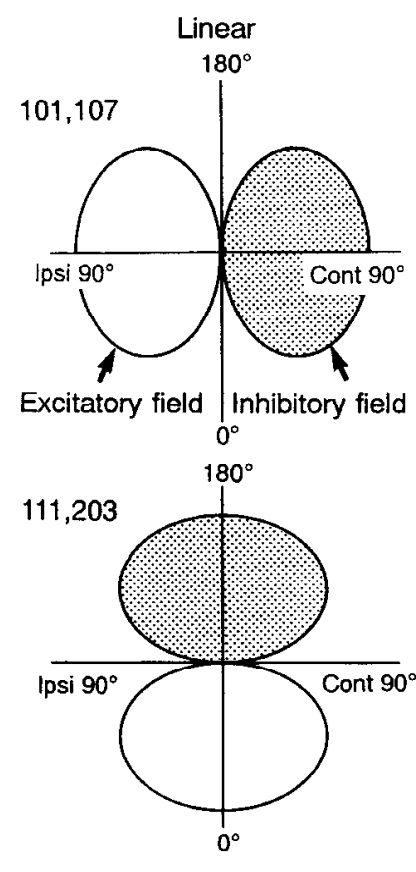
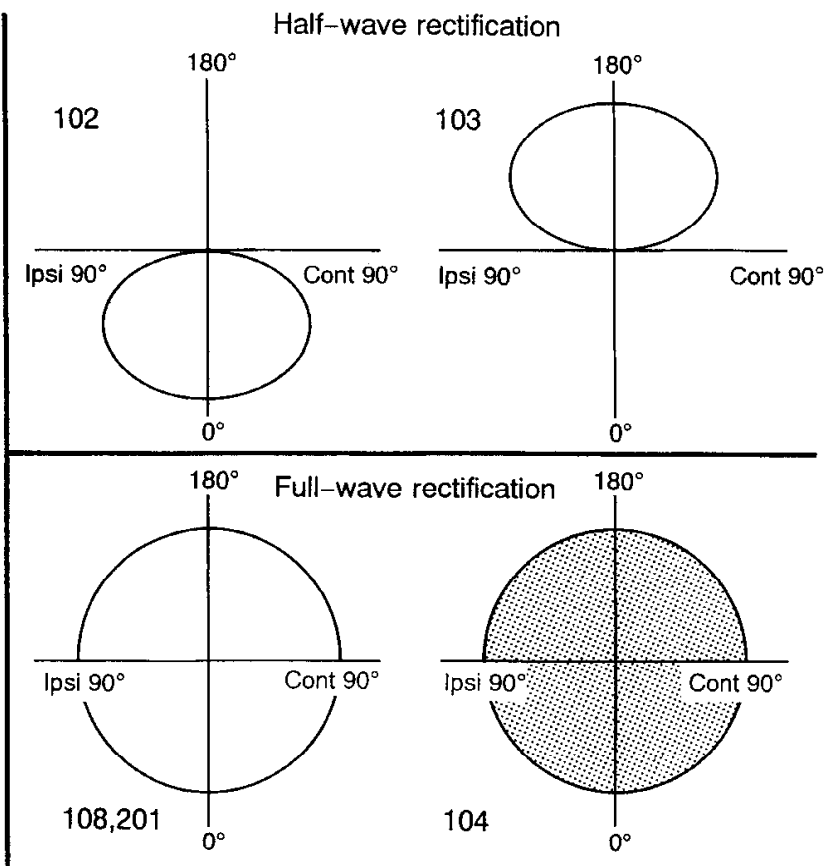

Figure 15. Schematic diagrams of directional properties of the nine nonspiking local interneurons. Shaded regions represent inhibitory directions; open regions represent excitatory directions. because spontaneous synaptic input was observed even in the absence of any wind stimulus. The corresponding activity of cercal afferents has also been observed by Westin (1979). Such noise at the input is a source of error in kernel estimation. If we take account of the noise, the MSE of $30-40 \%$ for the secondorder kernel is very reasonable. In the almost noise-free neurons, for example, horizontal cells in the vertebrate retina, MSE was close to 5-10\% (Sakai and Naka, 1987).

The second-order kernels predict the nonlinear part of the response arising from a static (nonmemory) nonlinearity such as squaring, rectification, saturation, and so on (Marmarelis and Marmarelis, 1978; Sakuranaga et al., 1986). The kernels can be used to construct a cascade model for the system, consisting of a static nonlinear element with one or two dynamic (memory) linear filters. The former is a cascade structure of Wiener type, a linear filter-static nonlinear filter cascade, and the latter is a cascade structure of Korenberg type in which a static nonlinearity is sandwiched between two linear filters (Korenberg and Hunter, 1986). For example, the type-C amacrine cells in the catfish retina produce a nonlinear response, an on-off transient depolarization to a step of light, whose second-order kernels have two depolarizing peaks on the diagonal and two off-diagonal, hyperpolarizing valleys (Sakuranaga and Naka, 1985a,b). Sakai and Naka (1987) showed that this second-order kernel can be reproduced by a band-pass linear filter followed by a squaring device. On the other hand, the second-order kernel of type- $\mathrm{N}$ amacrine cell is more complex, and is produced by further band-pass filtering of type-C amacrine cell responses.
In the cockroach, the second-order kernels showed a characteristic three-bar structure in which an elongated depolarizing peak on the diagonal is accompanied with off-diagonal hyperpolarizing valleys for $102,103,108$, and 201 . This kernel configuration was constructed by a band-pass linear filter-static nonlinear device-low-pass linear filter cascade, where the static nonlinearity is a squarer. The band-pass filter would simply reflect the mechanical property of cercal hair sensilla, whereas the low-pass filter represents the transfer at synapses between the cercal afferents and the interneurons. The squared output is therefore a reproduction of the cell's response. Either a multiplication of two sine waves or a full-wave rectification produces the second harmonic component that was seen in 104 and 201 when stimulated sinusoidally (Fig. 12C). As a full-wave rectifier is best approximated by a squarer, we reasonably concluded that this static nonlinear filter would represent a full-wave rectification of the signal. This LNL filter cascade, band-pass filterhalf-wave or full-wave rectification-low-pass filter, is equivalent to the theory of AM radio. The function of interneurons 104, 108 , and 201 as seen by their second-order kernels is thus to compute the power of wind stimuli at a particular frequency range. The second-order nonlinear component also comprises a major part in GIs 1 and 2 (Y. Kondoh and J. Okuma, unpublished observations). The full-wave rectification obviously represents the omnidirectional sensitivity of these interneurons (Westin, 1977; Dagan and Camhi, 1988).

On the other hand, the second-order nonlinearity in 102 and 103 was suggested to be a signal compression (a half-wave rec-

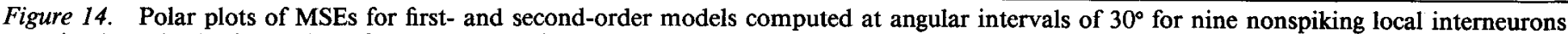

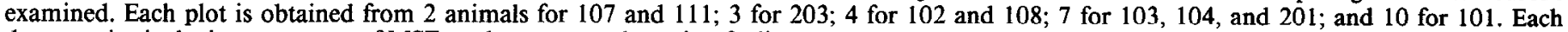

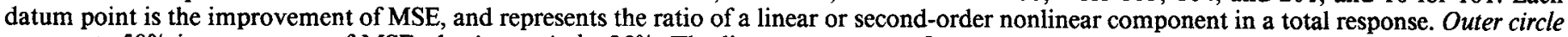

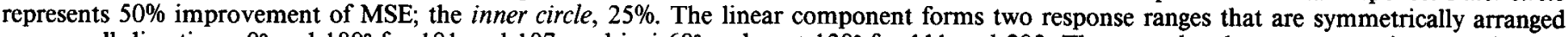

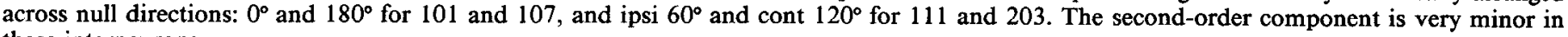
these interneurons. 
tification), and was modeled by a cascade of two filters: a bandpass linear filter and a static nonlinear filter. This filter configuration also accounts for the dynamics of the cercal receptors (Buño et al., 1981; Kondoh et al., 1991b). The first-order kernels from cercal filiform sensilla were biphasic, and closely matched the time differential of a pulse, whereas the second-order kernels were also differential with two diagonal peaks and two off-diagonal valleys (Kondoh et al., 1991b). The second-order nonlinearity is suggested to represent a half-wave rectification property of the wind receptors. All of the filiform hairs in the cockroach and cricket are deflected by wind preferentially in one plane (Nicklaus, 1965; Gnatzy, 1976). The sensory neurons are excited by only one of two directions of deflection of their filiform hairs, and the opposite direction elicits no response (Westin, 1979). Moreover, all the hairs within a given column have the same optimal excitatory direction (Dagan and Camhi, 1979). We therefore reasonably concluded that the interneurons with a half-wave-rectifier property receive excitatory input from a particular column of cercal hairs whereas those with a full-waverectifier property receive excitatory or inhibitory input from all columns.

\section{References}

Bacon JP, Murphey RK (1984) Receptive fields of cricket giant interneurons are related to their dendritic structure. J Physiol (Lond) 352:601-623.

Bodnar DA, Miller JP, Jacobs GA (1991) Anatomy and physiology of identified wind-sensitive local interneurons in the cricket cercal system. J Comp Physiol 168:553-564.

Boyan GS, Williams JLD, Ball EE (1989) The wind-sensitive cercal receptor/giant interneurone system of the locust, Locusta migratoria. IV. The non-giant interneurones J Comp Physiol 165:539-552.

Buño W, Monti-Bloch L, Mateos A, Handler P (1981) Dynamic properties of cockroach cercal "threadlike" hair sensilla. J Neurobiol 12: 123-141.

Burrows M (1980) The control of sets of motor neurones by local interneurones in the locust. J Physiol (Lond) 298:213-233.

Burrows M (1987) Parallel processing of proprioceptive signals by spiking local interneurons and motor neurons in the locust. J Neurosci 7:1064-1080.

Burrows M, Siegler MVS (1976) Transmission without spikes between locust interneurones and motoneurones. Nature 262:222-224.

Burrows M, Siegler MVS (1985) The organization of receptive fields of spiking local interneurons in the locust with inputs from hair afferents. J Neurophysiol 53:1147-1157.

Callec JJ (1974) Synaptic transmission in the central nervous system of insects. In: Insect neurobiology (Treherne JE, ed), pp 119-185. New York: North-Holland-American-Elsevier.

Camhi JM (1980) The escape system of the cockroach. Sci Am 243: 144-157.

Camhi JM, Levy A (1989) The code for stimulus directions in a cell assembly in the cockroach. J Comp Physiol 165:83-97.

Camhi JM, Nolen TG (1981) Properties of the escape system of cockroaches during walking. J Comp Physiol 142:339-346.

Camhi JM, Tom W (1978) The escape behavior of the cockroach Periplaneta americana. I. Turning response to wind puffs. J Comp Physiol 128:193-201.

Chappell RL, Naka K-I, Sakuranaga M (1985) Dynamics of turtle horizontal cell response. J Gen Physiol 86:423-453.

Dagan D, Camhi JM (1979) Response to wind recorded from the cercal nerve of the cockroach Periplaneta americana. II. Directional selectivity of the sensory neurons innervating single columns of filiform hairs. J Comp Physiol 133:103-110.

Daley DL, Camhi JM (1988) Connectivity pattern of the cercal-togiant interneuron system of the american cockroach. J Neurophysiol 60:1350-1368.

Egelhaaf M, Hausen K, Reichardt W, Wehrhahn C (1988) Visual course control in flies relies on neuronal computation of object and background motion. Trends Neurosci 11:351-358.
Gnatzy W (1976) The ultrastructure of the thread-hairs on the cerci of the cockroach Periplaneta americana L.: the intermolt phase. J Ultrastruct Res 54:124-134.

Gnatzy W, Tautz J (1980) Ultrastructure and mechanical properties of an insect mechanoreceptor: stimulus-transmitting structures and sensory apparatus of the cercal filiform hairs of Gryllus. Cell Tissue Res 213:441-463.

Hausen K (1981) Monocular and binocular computation of motion in the lobula plate of the fly. Verh Dtsch Zool Ges 1981:47-70.

Hausen K, Wehrhahn C. (1983) Microsurgical lesion of horizontal cells changes optomotor yaw responses in the blowfly Calliphora erythrocephala. J Exp Biol 219:211-216.

Jacobs GA, Miller JP, Murphey RK (1986) Integrative mechanisms controlling directional sensitivity of an identified sensory interneuron. J Neurosci 6:2298-2311.

Kämper $G$, Kleindienst $H$ (1990) Oscillation of cricket sensory hairs in a low-frequency sound field. J Comp Physiol 167:193-200.

Kanou M, Shimozawa T (1984) A threshold analysis of cricket cercal interneurons by an alternating air-current stimulus. J Comp Physiol 154:357-365.

Kobashi M, Yamaguchi T (1984) Local non-spiking interneurons in the cercus-to-giant interneuron system of crickets. Naturwissenschaften 71:154-155.

Kondoh Y, Hisada M (1986) Regional specialization in synaptic input and output in an identified local nonspiking interneuron of the crayfish revealed by light and electron microscopy. J Comp Neurol 251:334348.

Kondoh Y, Morishita H, Arima T, Okuma J (1989) Dynamics of a graded response in wind sensitive, nonspiking local interneurons of the cockroach. In: Neural mechanisms of behavior (Erber J, Menzel $R$, Pflüger HJ, Todt D, eds), abstract 21. Stuttgart: Thieme.

Kondoh Y, Morishita H, Arima T, Okuma J, Hasegawa Y (1991a) White noise analysis in a wind-sensitive, nonspiking interneuron of the cockroach. J Comp Physiol 168:429-443.

Kondoh Y, Arima T, Okuma J, Hasegawa Y (1991b) Filter characteristics of cercal afferents in the cockroach. J Comp Physiol 169: 653-662.

Korenberg MJ, Hunter JW (1986) The identification of nonlinear biological systems: LNL cascade models. Biol Cybern 55:125-134.

Krenz WD, Reichert H (1985) Lateralized inhibitory input to an identified non-spiking local interneuron in the crayfish mechanosensory system. J Comp Physiol 157:499-507.

Laurent $G$ (1988) Local circuits underlying excitation and inhibition of intersegmental interneurones in the locust. J Comp Physiol 162: 145-157.

Levine RB, Murphey RK (1980) Pre- and postsynaptic inhibition of identified giant interneurons in the cricket (Acheta domesticus). J Comp Physiol 135:269-282.

Marmarelis PZ, Marmarelis VZ (1978) Analysis of physiological system. New York: Plenum.

Marmarelis PZ, Naka K-I (1972) White-noise analysis of a neuron chain: an application of the white-noise theory. Science 175:12761278.

Matsumoto SG, Murphey RK (1977) The cercus-to-giant interneuron system of crickets. IV. Patterns of connectivity between receptors and the medial giant interneuron. J Comp Physiol 119:319-330.

Miller JP, Jacobs GA, Theunissen FE (1991) Representation of sensory information in the cricket cercal sensory system. I. Response properties of the primary interneurons. J Neurophysiol 66:1680-1689.

Naka K-I, Sakuranaga M, Ando Y-I (1985) White-noise analysis as a tool in vision physiology. In: Progress in clinical and biological research, Vol 176, Contemporary sensory neurobiology (Correia MJ, Perachio AA, eds), pp 307-322. New York: Liss.

Nicklaus R (1965) Die Erregung einzelner Fadenhaare von Periplaneta americana in Abhängigkeit von der Grösse und Richtung der Auslenkung. Z Vgl Physiol 50:331-362.

Reichert H, Plummer MR, Wine JJ (1983) Identified nonspiking local interneurons mediate nonrecurrent lateral inhibition of crayfish mechanoscnsory interneurons. J Comp Physiol 151:261-276.

Ritzmann RE (1981) Motor responses to paired stimulation of giant interneurons in the cockroach Periplaneta americana. II. The ventral giant interneurons. J Comp Physiol 143:71-80.

Ritzmann RE, Camhi MR (1978) Excitation of leg motor neurons by giant interneurons in the cockroach Periplaneta americana. J Comp Physiol 125:305-316. 
Ritzmann RE, Pollack (1981) Motor responses to paired stimulation of giant interneurons in the cockroach Periplaneta americana. I. The dorsal giant interneurons. J Comp Physiol 143:61-70.

Robertson RM, Pearson KG (1983) Interneurons in the flight system of the locust: distribution, connections, and resetting properties. J Comp Neurol 215:33-50.

Sakai HN, Naka K-I (1987) Signal transmission in the catfish. V. Sensitivity and circuit. J Neurophysiol 58:1329-1350.

Sakuranaga M, Naka K-I (1985a) Signal transmission in the catfish retina. I. Transmission in the outer retina. J Neurophysiol 53:373389.

Sakuranaga M, Naka K-I (1985b) Signal transmission in the catfish retina. II. Transmission to type $\mathrm{N}$ cell. J Ncurophysiol 53:390-410.

Sakuranaga M, Ando Y-I, Naka K-I (1986) Dynamics of ganglioncell response in the catfish and frog retinas. J Gen Physiol 90:229259.

Shimozawa T, Kanou M (1984) The aerodynamics and sensory physiology of range fractionation in the cercal filiform sensilla of the cricket Gryllus bimaculatus. J Comp Physiol 155:495-505.

Siegler MVS (1984) Local interneurons and local interactions in arthropods. J Exp Biol 112:253-281.
Siegler MVS, Burrows M (1983) Spiking local interneurons as primary integrators of mechanosensory information in the locust. J Neurophysiol 50:1281-1295.

Westin J (1979) Responses to wind recorded from the cercal nerve of the cockroach Periplaneta americana. I. Response properties of single sensory neurons. J Comp Physiol 133:97-102.

Westin J, Ritzmann RE (1982) The effect of single giant interneuron lesions on wind-evoked motor responses in the cockroach Periplaneta americana. J Neurobiol 13:127-139.

Westin J, Langberg JJ, Camhi JM (1977) Response of giant interneurons of the cockroach Periplaneta americana to wind puffs of different directions and velocities. J Comp Physiol 121:307-324.

Westin J, Ritzmann RE, Goddard DJ (1988) Wind-activatcd thoracic interneurons of the cockroach. I. Responses to controlled wind stimulation. J Neurobiol 19:573-588.

Wohlers DW, Huber F (1978) Intracellular recording and staining of cricket auditory interneurons (Gryllus campestris L., Gryllus bimaculatus DeGeer). J Comp Physiol 127:11-28. 